Aus dem pathologisch-histiologischen Institute in Wien.

\title{
Beiträge zur pathologischen Anatomie der Blennorrhoe der männlichen Sexualorgane.
}

\section{Die ehronische Urethritis posterior und die chronische Prostatitis.}

\author{
Von \\ Dr. Ernest Finger, \\ Docenten in Wien.
}

(Hierzu die Tafeln I. bis III.)

Die vorliegende Arbeit stellt sich als Fortsetzung einer in diesem Archiv (s. Archiv für Dermatologie und Syphilis 1891. Ergänzungsheft I) niedergelegten Arbeit dar.

Wie schon dort berichtet, war mir durch die grosse Liberalität des Herrn Professor Weichselba um das Material für Untersuchungen über die pathologische Anatomie und Histiologie der männlichen Urethralblennorrhoe zugänglich gemacht worden. Die systematische Untersuchung der männlichen Genitalien von circa 120 Leichen ergab in 31 Fällen auf chronische Urethritis zu beziehende Veränderungen. In 24 Fällen sassen diese Veränderungen in der Pars pendula, Bulbus, Pars membranacea und wurde über diese Fälle im ersten Theile der Arbeit ausführlich berichtet.

In elf Fällen ergaben sich Veränderungen im hinteren Theile der Harnröhre. 
Diese zu schildern ist Aufgabe der vorliegenden Zeilen die sich mit der Urethritis chronica posterior und der diese complicirenden chronischen Prostatitis beschäftigen werden.

Von diesen elf Fällen sass der chronisch-entzündliche Process in Pars anterior und Pars prostatica . . 5mal Pars prostatica allein ..... . . $6 \mathrm{mal}$

Sa. 11

Ich übergehe nun zunächst zur detaillirten Schilderung dieser Fälle und werde an diese dann die entsprechenden epikritischen Bemerkungen anfügen.

Erwähnen will ich nur noch, dass die den möglichst frischen Leichen entnommenen Präparate in Alkohol oder Müller'scher Lösung gehärtet, mittelst Mikrotom in Schnitte zerlegt, mit Carmin, Hämatoxylin, Occedin, die auf Mikroorganismen untersuchten Schnitte nach $K \ddot{u h n e}$ 's und Löffler's Methylenblaumethode gefärbt wurden.

Die nun anzuführenden Fälle sind systematisch, nach der Intensität und Extensität des Processes angereiht. Die der fortlaufenden Zahl des Falles in Klammern beigesetzte Zahl bezieht sich auf den ersten Theil und die dortselbst geschilderten Veränderungen in der Pars anterior, die ich hier nur im Resumé wiedergebe.

Fall I. (I) H. Th., 22 Jahre alt, Kellner, starb 8. März 1890 an Lungentuberculose.

Die Section ergab die Schleimhaut im Bereiche der Pars pendula fleckweise rauh, wie gestichelt oder leicht excoriirt.

Das Caput gallinaginis anffallend vergrössert, die Schleimhaut desselben und der Pars prostatica wie gestichelt und aufgelockert.

Druck auf die Prostata entleert ein dickes, milchweisses, an epithelialen Zellen und Amyloidkörperchen reiches Secret.

Die mikroskopische Untersuchung ergab

In der Pars anterior:

proliferation und Desquamation des noch als Cylinderepithel erhaltenen Epithels. Oberfläcbliche, subepitheliale, aus mononuclearen und epitheloiden Zellen bestehende Infiltration, die um Lacunen und Drüsen etwas tiefer dringt, insbesondere als Begleiterin der Drüsenausführungsgänge der Littre'schen Drüsen aber bis in das Corpus cavernosum vordringt. Catarrhalische Desquamation und Proliferation der Lacunen und Drüsenausführungsgänge. Die Littre'schen Drüsen bis auf leichte interstitielle Infiltration unverändert. Minimale Production polynuclearer Leu- 
cocyten, Gonococcen spärlich in den obersten Epithellagen der Mucosa und Drüsen. (Details siehe I. Theil, Fall I.)

In der Pars posterior:

Epithel. a) Der Urethra: Dieses soweit erhalten, stellt sich als niederes Cylinderepithel dar, aus einer oberen Schichte meist zerworfener und in Abstossung begriffener Cylinderepithelien, einer unteren Schichte cubischer E'rsatzzellen bestehend.

b) Am Caput gallinaginis, dessen Epithel zum grössten Theile fehit, stellen die Reste ein flaches, aus zwei Reihen niederer Epithelien bestehendes Plattenepithel dar.

Subepitheliale Bindegewebe: a) der Urethra. Das subepitheliale Bindegewebe der Urethra ist bis in bedeutende Tiefe von einem gleichmässig dichten, nach unten ganz scharf absetzenden Infiltrate durchsetzt, das aus dicht gedrängten, mononuclearen Rundzellen und epitheloiden Zellen besteht. An einigen, insbesondere oberflächlichen Stellen finden sich Inseln, wo dieses Infiltrat einer Necrose anheimgefallen ist, der Contour der das Infiltrat constituirenden Zellen ist noch erhalten, diese selbst aber haben ihre Tinctionsfähigkeit eingebüsst, sind schwach diffus oder gar nicht gefärbt. In dieses äberall ziemlich gleich breite bandartige und auch fast überall gleich dichte Infiltrat sind die Drüsen der Urethra eingesprengt.

b) Caput gallinaginis. Dasselbe breite, bandartige, stellenweise in Necrose übergegangene dichte, aus mononuclearen und epitheloiden Zellen bestehende Infiltrat, wie im subepithelialen Bindegewebe der Urethra, zieht sich auch als gleichmässiges Band direct subepithelial im Bogen über das ganze Caput gallinaginis. Dasselbe bedingt $z$ weifellos die schon makroskopisch auffallende Vergrösserung des Caput gallinaginis.

Drïsen: a) der Urethra. Die oberfiächlichen seichten Lacunen und kleinen Drüsen der Urethra sind alle in das Infiltrat des subepithelialen Bindegewebes eingebettet, das Epithel derselben stellt sich als proliferirendes Cylinderepithel dar, das insbesondere in den Drüsen, deren Lumen durch das Infiltrat sehr eingeengt erscheint, dieses ganz ausfüllt.

b) Caput gallinaginis. Die Drüsen des 'Caput gallinaginis, sowie der Utriculus sind fast ausnahmslos in derselben Weise verändert, dass das niedere Cylinderepithel proliferirt und desquamirt, wodurch das oft recht weite Lumen der Drüse sowohl von zahlreichen abgestossenen Epithelzellen als von vielen oft grossen Amyloidenkörperchen erfüllt erscheint. Die oberflächlichen Drüsen und Drüsenantheile sitzen mitten in dem oben beschriebenen Infiltrate, erscheinen dann durch dasselbe vielfach comprimirt, ihr Lumen verengt, oft bis auf einen kleinen Spalt reducirt. Aber entlang der Wand der meisten Drüsen, dicht unter dem Epithel pflegt das oberflächliche Infiltrat sich in Form eines nach unten zu schmäler und schütterer werdenden Bandes längst der Drüsen fortzusetzen, in die Zotten derselben einzudringen, diese dadurch kolbig $z$ u vergrössern. Dieses Infiltrat besteht wie im Subepithel, so auch tiefer 
ausschliesslich aus mononuclearen Rundzellen und epitheloiden, spärlichen Spindelzellen.

c) Prostata. Die tiefer liegenden Drüsen der Prostata sind normal. Die oberflächlicheren weisen reichliche Proliferation des Epithels, Erfüllung des Lumens mit abgestossenen Epithelien und Amyloiden auf. Aber auch das Infiltrat des subepithelialen Bindegewebes hat, längs der Ausführungsgänge und oberflächlicheren I)rüsen seinen Weg in die Tiefe gefunden und zahlreiche Prostatadrüsen zeigen eine oft noch recht bedeutende und dichte Infiltration des zottenförmig vorspringenden. intertubulösen Bindegewebes und der Wand mit mononuclearen Rundzellen und epitheloiden Zellen.

d) Ductus ejaculatorii. Beide Ductus ejaculatorii münden als schmale, unregelmässige Spalten in dem dichten Infiltrate des subepithelialen Bindegewebes am Caput gallinaginis. Innerhatb dieses Infiltrates fehlt ihr Epithel, die Wand wird ausschliesslich von dem dichten Infiltrate gebildet. Unterhalb des Infiltıates zeigen sie ein cubisches, in Proliferation und Abstossung begriffenes Epithel. Die Wand der Ductus ejaculatorii zeigt deren ganzer Länge nach, soweit sie durch die Prostata $z u$ verfolgen sind, ein ziemlich breites, aus epitheloiden und mononuclearen Rundzellen, aber auch viel Spindelzellen bestehendes lnfiltrat, das sich als Fortsetzung des oben beschriebenen subepithelialen Infiltrates darstellt. Dabei erscheint das Lumen der Ductus nicht gleichmässig, sondern zeigt abwechselnd Verengerungen und spindelförmige Auftreibungen.

Resumé. Desquamativer Catarrh der Urethra. Epithel des Caput: gallinaginis, niederes Plattenepithel. Rein desquamativer Catarrh der Lacunen und Drüsen der Urethra, des Utriculus, der Drüsen des Caput. gallinaginis und zahlreicher Prostatadrüsen.

Dichte und tiefgreifende, aus mononuclearen Rundzellen und epitheloiden Zellen bestehende Infiltration des subepithelialen Bindegewebes. von Urethra und Caput gallinaginis.

Dieses Infiltrat setzt sich als periglanduläre Wandinfiltration auf die Drüsen des Caput gullinaginis und zahlreiche Prostatadrüsen fort.

Die Ductus ejaculatorii, deren Mündung durch das Infiltrat verengt, zeigen Proliferation und Desquamation ihres Epithels, Infiltration ihrer Wand, ihrer ganzen Länge nach durch ein sich auch aus dem subepithelialen Bindegewebe fortsetzendes Infiltrat.

Fall II. M. M., 26 Jahre alter Schuster, starb am 31. März 1890. an Tuberculose.

Die Section ergab in der Pars anterior urethrae keine auffälligen Veränderungen, dagegen erscheint in der Pars posterior das Caput gallinaginis vergrössert, an dessen Kuppe eine umschriebene abschliffartige: Depression.

Die milkroskopische Untersuchung ergab:

Epithel. a) Urethra: Das Epithel der Harnröhre ist allüberall ein normales, festgefügtes Cylinderepithel mit einer oberen Reihe palis- 
sadenförmig angeordneter Cylinderepithelien und einer unteren Reihe Ersatzzellen.

b) Caput gallinaginis. Auch am Caput gallinaginis erscheint das. Epithel zumeist als normales Cylinderepithel.

Nur jene Stelle, die sich makroskopisch als Depression erweist, trägt ein aus vielen Lagen polyedrischer Zellen bestehendes Plattenepithel, das ganz unvermittelt in das Cylinderepithel übergeht.

Subepitheliales Bindegewebe. Das subepitheliale Bindegewebe sowohl a) der Urethra, als b) des Caput gallinaginis ist locker, wellig, in seinen obersten Lagen ist dasselbe von einem lockeren Infiltrate durchsetzt, das unter dem Cylinderepithel aus mononucleareu Rundzellen und epitheloiden Zellen besteht, während es unter dem Plattenepithel an der Kuppe des Caput gallinaginis vorwiegend spindelförmige Kerne, als mehr den Charakter derberen Bindegewebes darbietet.

Drüsen. Die Drüsen: a) der Urethra zeigen keine Veränderungen, dagegen sind die oberfächlichen Drüsen des b) Caput gallinaginis, insbesondere der Utriculus bedeutend verändert. Diese zeigen zunächst ein kleinzelliges, meist aus mononuclearen Rundzellen, weniger epitheloiden Zellen bestehendes Infiltrat, das weniger periglandulär, vorwiegend. interstitiell ist. Dieses interstitielle Infiltrat führt zu Verlängerung, Verbreiterung des intertubulösen Bindegewebes, das sich auf Durchschnitten als zwischen den Drüsentubulis gelegene, diese trennende, von Drüsenepithel gedeckte zottenartige Vorsprünge präsentirt. Diese verlängerten und verbreiterten Zotten legen sich an einander, ja sie scheinen nach Verlust des Epithels zu verwachsen, wodurch die von denselben begrenzten Drüsentubuli gegen das Lumen der Drüse abgeschlossen werden, sich auf Durchschnitten wie Drüsenacini darstellen. Diese abgeschnürten Tubuli zeigen nun auch wieder Proliferation des Epithels und führen oft reichlich Corpuscula amylacea, so dass ihr Lumen von diesen und den Epithelzellen gänzlich erfüllt ist, während die Infiltration des intertubulösen Bindegewebes auch zur Verengerung des Lumens beiträgt.

Diese so beschriebene Veränderung zeigt der Utriculus durchgehends, wir finden dieselbe aber auch an zahlreichen Drüsen des Caput. gallinaginis, während andere völlig normal erscheinen.

c) Die Drüsen der Prostat a selbst jedoch sind durchwegs normal.

d) Die Ductus ejaculatorii zeigen während ihres Verlaufes durch das Caput gallinaginis leichte periglanduläre und interstitielle, insbesondere in die Zotten localisirte Infiltration mit mononuclearen Rundzellen und Spindelzellen. Das Epithel führt besonders in der obersten Schichte reichlich gelbes, grobkörniges Pigment, das insbesondere im. Epithel der in der Wand der Ductus ejaculatorii sitzenden einfachen Drüsentubuli reichlich ist. Das Lumen der Ductus ejaculatorii, nicht aber der Drüsentubuli führt reichlich die Elemente des Sperma; zahlreiche Spermatozoen, epitheliale Elemente und grobkörniges gelbes Pigment. 
Res u mé. Oberfiächliche chronische Entzündung des subepithelialen Bindegewebes mit Bildung einer circumscripten, von Plattenepithel gedeckten Schwiele an der Kuppe des Caput gallinaginis. Catarrhaliseh desquamative und interstitielle Entzündung des Utriculus und zahlreicher Drüsen am Capnt gallinaginis. Periglanduläre Infiltration des Endstückes der Ductus ejaculatorii.

Fall III. Z. H., 20 Jahre alt, Taglöhner, stąrb am 18. März 1890 an Tuberculose.

Die Section zeigte in der Pars anterior urethrae keine auffälligen Veränderungen, dagegen erscheint in der Pars posterior die Schleimhaut der Urethra um das Caput gallinaginis bis nahe an das Ostium internum aufgelockert. Das Caput gallinaginis erscheint sehr bedeutend vergrössert. Druck auf die Prostata entleert aus den Ausführungsgängen der prostatischer Drüsen ein dickes, milchiges Secret, das unter dem Mikroskop an Epithelzellen sehr reich ist, nur spärliche Amyloide, keine Eiterkörperchen führt.

Die mikroskopische Untersuchung ergab:

Epithel. a) Urethra: Das Epithel der Harnröhre stellt sich alliiberall als Cylinderepithel dar, dessen oberste Schichte der Cylinderzellen zerworfen, theilweise abgestossen, theilweise schleimig degenerirt ist. Unter derselben eine bis zwei Reihen polygonaler Ersatzzellen.

b) Caput gallinaginis. Auch hier finden wir djeselben Veränderungen. Desquamative und schleimige Degeneration des Cylinderepithels, leichte Verbreiterung der Ersatzzellenschichte.

Subepitheliales Bindegewebe. a) Urethra. Die obersten Schichten des subepithelialen Bindegewebes der Urethra zeigen in der ganzen Ausdehnung um das Caput gallinaginis in gegen die Blase zu abnehmender Intensität ein Auswachsen zu theils spitzen, theils kolbig, knopfförmig anschwellenden Excrescenzen. deren Substrat aus zwei Factoren gebildet wird. Einmal aus einem ziemlich lockeren Bindegewebe, das in einer von oben nach unten zu abnehmenden Intensität von einem im Ganzen recht lockeren, schütteren, zelligen Infiltrate durchsetzt wird, welches der Hauptmasse nach aus mononuclearen Rundzellen und epitheloiden Zellen gebildet ist, denen aber in nicht unbedeutender Menge auch Spindelzellen beigemengt sind. Dann aber ist an der Bildung dieser papillaren Excrescenzen noch ein zweiter Factor betheiligt: eine grosse Zahl auffallig weiter Gefässe, die mit oft noch bedeutendem Durchmesser bis in die obersten Schichten des subepithelialen Bindegewebes hinau nachzuweisen sind, keine auffalligere Infiltration der Wand darbieten und durch ihre grosse Zahl, ihr grosses Caliber dem subepithelialen Bindegewebe viel Aehnlichkeit mit Schwellgewebe geben.

b) Caput gallinaginis. Das subepitheliale Bindegewebe des Caput gallinaginis zeigt nirgend die Tendenz zur Bildung papillarer Excrescenzen. Ueberall zieht es glatt dahin. Wohl aber ist dasselbe auch insbesondere in den obersten Schichten von einem zelligen Infiltrate durch- 
setzt, das aus mononuclearen Rundzellen, epitheloiden und recht zahlreichen Spindelzellen besteht. Dieses Infiltrat ist nur als dünnes Band in den obersten Schichten des subepithelialen Bindegewebes nachzuweisen. Nur an den beiden Ductus ejaculatorii setzt sich dasselbe auch hier subepithelial als dünner Schlauch, dieselben umschliessend, in die Tiefe fort, begleitet dieselben während des ganzen Verlaufes durch das Caput gallinaginis, um an Dichte allmälig abnehmend, in der Tiefe völlig anfzuhören.

Drüsen. a) Urethra. Die in Folge der Bildung der oben genannten papillaren Excrescenzen sehr tiefen Buchten und Lacunen der Urethra sind mit desquamirtem, schleimig degenerirendem Cylinderepithel ausgezleidet. Die oberfächlichen Drüsen der Urethra sitzen zum Theil in dem subepithelialen Infiltrate. Auch sie weisen Proliferation ihres Epithels oft bis zur völligen Erfüllung des Lumens mit Epithelzellen auf, führen nur spärliche Amyloide.

b) Caput gallinaginis. Der Utriculus nnd zahlreiche Drüsen des Caput gallinaginis erscheinen völlig normal. Die oberflächlichsten Drüsen dagegen, die im oberflächlichen subepithelialen Infiltrate sitzen, ebenso aber auch zahlreiche tiefere Drüsen, die keine Veränderungen ihrer Wand, des sie umgebenden Bindegewebes darbieten, zeigen Proliferation des Epithels, Erfüllung des Lumen mit proliferirten, abgestossenen Epithelzellen.

c) Prostata. Auch hier finden wir analoge Veränderungen. Ein grosser Theil der Prostatadrüsen völlig normal, in anderen dagegen, ohne Veränderung der Wand, des umgebenden Bindegewebes, Erfüllung des Lumen mit proliferirten, abgestossenen Epithelzellen. Dabei fällt die äusserst geringe Zabl von Amyloiden auf.

Resumé. Oberfächliche, chronische, in der Urethra zur Bildung papillarer Excrescenzen führende Entzündung des subepithelialen Bindegewebes, die nur längs der beiden Ductus ejaculatorii in die Tiefe dringt. Rein desquamativer Catarh zahlreicher Drüsen der Urethra, des Caput gallinaginis und der Prostata.

Fall IV. (XV) S. J., 34 Jahre alt, Taglöhner, starb am 26. Jänner 1890 an Gehirnblutung.

Die Section zeigte in der vorderen Harnröhre, an derer unteren Wand eine flache, leicht deprimirte, aber nicht stricturirende Narbe, in deren Umgebung die Schleimhaut fein gewulstet, wie drusig uneben erscheint.

Zu beiden Seiten des Caput gallinaginis erscheint die Schleimhaut papillar aufgelockert.

Die mikroskopische Untersuchung ergab:

In der Pars anterior: Chronische, zur Bildung von Fxcrescenzen führende, im Centrum in eine ganz oberflächliche, nicht stricturirende Schwiele ausgehende Entzündung des subepithelialen Bindege-

Ergänzunģhefte z. Archiv f. Dermatol. u. Syphil. 1893. 
webes. Ueber der Schwiele Umwandlung des Cylinder- in Plattenepithel. (Details siehe I. Theil, Fall XV.)

In der Pars posterior:

Epithel. a) Urethra. Das Epithel der Hararöhre ist überall, auch dort, wo es die noch zu beschreibenden papillaren Excrescenzen des subepithelialen Bindegewebes deckt, ein Cylinderepithel, dessen oberste Schichte der Cylinderzellen theils in Abstossung, theils in schleimiger Degeneration begriffen ist, während die Schichte der Ersatzzellen auf 3-4 Lagen verbreitert erscheint.

b) Caput gallinaginis. An den Seiten des Caput gallinaginis, soweit die papillaren Wucherungen des subepithelialen Bindegewebes reichen, ist das Epithel, analog dem der Urethra, ein Cylinderepithel mit verbreiterter Ersatzzellenschichte. An der Kuppe des Caput gallinaginis, wo die Excrescenzen aufhören, das Bindegewebe in glattem Bogen über die Kuppe hinzieht, erscheint das Epithel als Plattenepithel, indem mehreren Lagen polygonaler eine bis zwei Reihen Plattenepithelien aufruhen.

Subepitheliale Bindegewebe. a) Urethra. Das subepitheliale Bindegewebe der Harnröhre zeigt an der oberen Wand nur eine leichte aus mononuclearen Rundzellen, wenigen epitheloiden, reichlichen Spindelzellen bestehende Infiltration des glatten, lockeren, welligen Bindegewebes. Je weiter an den Seiten herunter, desto mehr nimmt diese Infitration an Dichte zu und an den Seitentheilen, in der Rinne zwischen Urethra und Caput gallinaginis erscheint das subepitheliale Bindegewebe dicht und tiefer infiltrirt. Gleichzeitig zeigt das Bindegewebe eine Reihe von theils einfach papillaren, theils kolbig angeschwollenen Excrescenzen, die ganz aus kleinzellig infiltrirtem Bindegewebe bestehend, Drüsenbläschen halten. Diese Excrescenzen nehmen von der Seite nach der unteren Wand der Harnröhre an Höhe zu, sind dort, wo die Falte zwischen Harnröhre und Caput gallinaginis an tiefsten, also am Grunde dieser Falte oder Rinne am höchsten.

b) Caput gallinaginis. An den Seitentheilen desselben finden wir dieselben papillaren und kolbigen Bindegewebs-Excrescenzen, die gegen die Kuppe allmälig niederer, kleiner werden und ehe sie die Kuppe des Caput gallinaginis erreichen, völlig versehwinden, so dass das Bindegewebe über die Kuppe in glattem Bogen fortzieht. Auch hier zeigt das Bindegewebe dieselbe Infiltration mit mononuclearen Rundzellen und Spindelzellen, die in den Excrescenzen am dichtesten, an der Kuppe des Caput gallinaginis nur gering und locker ist.

Drüsen. a) Urethra. Sowohl die overfächlichen, als die in die Excrescenzen eingebetteten Drüsen, sowie die seichten Lacunen zeigen Proliferation, Desquamation, theilweise schleimige Degeneration des Epithels, periglanduläre Infiltration.

b) Caput gallinaginis. Von den Drüsen desselben zeigt nur der Utriculus auffallendere Veränderungen. Diese bestehen in leichter periglandulärer Infiltration, in intensiver Infiltration der Zotten mit mononuclearen und Spindelzellen. Durch diese Infiltration werden die Zotten 
verlängert, verbreitert, kolbig. Das Epithel zeigt, eine Verbreiterung der Ersatzzellen auf 3-4 Lagen ausgenommen, keine Veränderung.

Die übrigen Drüsen des Caput gallinaginis zeigen keine Verände-

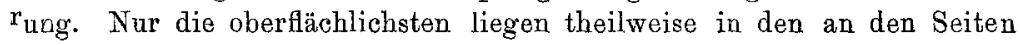
des Caput gallinaginis beschriebenen papillären Wucherungen, sind dann periglandulär infiltrirt, ihr Lumen verengt, mit desquamirtem Epithel exfüllt.

c) Prostata. Die Drüsen der Prostata sind durchwegs normal.

Zu erwähnen ist noch ein Befund an den Ductus ejaculatorii Beide Ductus ejaculatorii sind leicht periglandulär infiltrirt. Das Infiltrat besteht aus mononuclearen Rundzellen und Spindelzellen. Während dieses Infiltrat auch in die Zotten des linken Ductus ejaculatorius eindringt, diese verlängert und verbreitert, finden wir am rechten Ductus ejaculatorius auch periglanduläre Infiltration, aber auch bedeutende Erweiterung des Lumens mit Verstreichen der Zotten, so dass das Lumen desselben anstatt des normalen, vielfach buchtigen einen völlig runden Querschnitt darbietet.

Resumé. Chronische Entzündung nait Bildung papillarer Excrescenzen an der Urethra posterior und den Seitentheilen des Caput gallinaginis. Periglanduläre chronische Entzündung des Utriculus und der Ductus ejaculatorii mit Erweiterung des Lumen des rechten.

Fall V. R. F., 28 Jahre alt, Diener, starb am 19. Jänner 189 an Tuberculose.

Die Section ergab in der Pars anterior nichts Abuormes, dagegen erscheint in der Pars posterior das Caput gallinaginis auffällig vergrössert, blutarm, gelblich verfärbt. An der Kuppe ein schwarzer, submucös sitzender hirsekorngrosser Punkt. Die Schleimhaut der Urethra erscheint um das Caput gallinaginis herum und hinter demselben bis knapp an den Uebergang in dje Blasenschleimhaut aufgelockert, wie gestichelt.

Die mikroskopische Untersuchung ergab:

Epithel: a) Urethra. Das Epithel der Schleimhaut der ganzen Pars prostatica bis an die Blase ist ein proliferirendes, desquamirendes Cylinderepithel, das aus einer obersten Schichte zum Theil noch palissadenförmig angeordneter, meist aber zerworfener, locker aufsitzender oder abgestossener Cylinderepithelien und mebreren Lagen polyedrischer oder cubischer Ersatzzellen besteht.

b) Caput gallinaginis. Das Epithel des Caput gallinaginis ist ein festgefügtes Cylinderepithel, das aus einer oberen Schichte palissadenförmig angereihter, sehr niederer Cylinderepithelien besteht, die einer. einfachen oder doppelten Reihe polyedrischer Ersatzzellen aufruhen.

Subepitheliales Bindegewebe. a) Lrethra. Das subepitheliale Bindegewebè der ganzen Schleimhaut der Pars prostatica bis an die Blase zeigt in seiner obersten Schichte eine grosse $Z a h l$ dicht bei ein: ander stehender, spitzer oder kolbiger papillärer Excrescenzen, die von dem oben beschriebenen, desquamirender Cylinderepithel überzogen; das 
als schmales Band Wellenberge und Wellenthäler gleichmässig contourirt. Das diese papillaren Excrescenzen bildende Bindegewebe erscheint im Gegensatz zu der lockeren, welligen Beschaffenheit der tieferen Schichten des submucösen Bindegewebes mehr straff, zeigt mässige Infiltration, die aber nur aus spärlichen Rundzellen, meist ans spindelförmigen, älteren Bindegewebszellen besteht.

b) Caput gallinaginis. Die obersten Bindegewebszäge des subepithelialen Bindegewebes am Caput gallinaginis präsentiren sich gleichfalls als Züge eines ziemlich straffen, derben Bindegewebes, das aber noch an Rundzellen, insbesondere Spindelzellen ziemlich reich ist und glatt über den Conus des Caput gallinaginis hinzieht.

Drüsen. a) Urethra. Die oberfächlichen Drüsen und seichteu Lacunen der Urethra, deren erstere in das infiltrirte Bindegewebe ein. gebettet erscheinen, zeigen Proliferation ihres Epithels, àas im Verein mit Amyloiden das Lumen mancher Drüse oder Lacune völlig erfüllt.

b) Caput gallinaginis. Die oberflächlichen, im schwieligen Bindegewebe sitzenden. Drüsen zeigen Verengerung ihres Lumen, das von proliferirtem Epithel oder nur von Amyloiden erfüllt wird.

Auffällige und interessante Veränderungen zeigen: c) Der Utriculus, dessen Lumen ganz von einem grossen, schon makroskopisch als sehwarzer Punkt an der Kuppe des Caput gallinaginis sichtbares Corpus amylaceum ausgekleidet wird. Das Epithel des Utriculus zeigt keine Veränderungen, wohl aber das periglanduläre Bindegewebe. Sowohl die nächsten Schichten des den Utriculus einschliessenden Bindegewebes, als das intertubuläre Bindegewebe des Utriculus pràsentirt sich als strafferes, schwieligeres, aber von Spindelzellen und Rundzellen noch ziemlich reichlich durchsetztes Bindegewebe, das als directe Fortsetzung des in analoger Weise veränderten subepithelialen Bindegewebes entlang den Wänden des Utriculus erscheint.

Insbesondere auffällig ist aber die Verïnderung an $l$ ) den Ductus ejaculatorii. Auch hier erscheint das die Ductus ejaculatorii einschliessende Bindegewebe, so wie das intertubulöse, die Tubuli der Ductus ejaculatorii trennende Bindegewebe straffer, derber, aber immer noch reich an runden und spindelförmigen Zellen. Diese Veränderung der Wand der Dactus ejaculatorii ist während deren Verlaufs durch das Caput gallinaginis zu verfolgen.

Während des Verlaufes durch die Prostata zeigt das die Wand, die nächste Umgebung der Ductus ejaculatorii bildende Bindegewebe lockere, normale Beschaffenheit, das Bindegewebe der intertubulösen Zotten jedoch bleibt während des ganzen Verlaufes straffer, derber, reich an Spindelzellen und Rundzellen. Auch hier erscheint diese schwielige Veräıderung des Bindegewebes als directe Forisetzung der oberfächlichen schwieligen Degeneration der obersten Schichten des subepithelialen Bindegewebes des Caput gallinaginis.

Das Epithel der Iuctus ejaculatorii erscheint normal, als obere Schichte niederer palissadenförmig angereihter, fast cubischer Cylinder- 
epithelien und einer oder zwei Lagen polygonaler Ersatzzellen, auffallend ist jedoch die grosse Zahl körniger, gelber Pigmentklumpen, die sich insbesondere in der obersten Schichte des Epithels finden.

Auffällig ist endlich noch ein Umstand. Die Ductus ejaculatorii sind de norma keine einfachen Schläuche. Die Wand derselben hat zahlreiche, einfache oder verzweigte Divertikel, die zur Längsachse schief mit ihrer Längsrichtung in der Richtung nach der Mündung der Ductus ejaculatorii zu verlaufen. So erscheint auch auf Schiefschnitten der Ductus ejaculatorius nicht als Gang, sondern jeder einzelne scheint aus mehreren Gängen zu bestehen, indem man neben dem Querschnitt des 1)uctus ejaculatorius und um diesen eine Reihe von Querschnitten der Divertikel gruppirt sieht. Alle diese Divertikel sind von demselben Epithel wie der Ductus ejaculatorius ausgekleidet. In unserem Falle nun erscheinen fast sämmitliche Divertikel beider Ductus ejaculatorii, insbesondere im Bereich des Caput gallinaginis, aber auch im Verlaufe durch die Prostata mit den Elementen der Sperma, Epithelzellen, zahlreichen Spermatozoen, zahlreichen, gelben Pigmentschollen oft dicht erfüllt.

c) Prostata. Die Drüsen derselben sowie das interacinöse Gewebe völlig normal.

Res u m é. Chronische Entzündung des subepithelialen Bindegewebes am Wege zur Schwielenbildung schon ziemlích weit vorgeschritten, mit Bildung papillarer Excrescenzen in den obersten Lagen des subepithelialen Bindegewebes, auf der Schleimhaut der Urethra prostatica, mit Bildung straffen, glatten Bindegewebes am Caput gallinaginis.

Chronische, auf dem Wege zur Schwielenbildung ziemlich weit vorgerückte Entzündung des Bindegewebes der Wand des Utriculus und beider Ductus ejaculatorii. Die Divertikel beider Ductus ejaculatorii dicht mit Sperma erfüllt.

Fall VI. E. J., 44 Jahre alt, Gerichtsdiener, starb am 27. October 1889 an Lungentuberculose.

Die Section ergab:

In der Pars pendula urethrae drei quer verlaufende, leistenförmige, nicht stricturirende Narben. Das Caput gallinaginis vergrössert, Druck auf die nicht vergrösserte Prostata entleert aus den Ausführungsgängen der Prostatadrüsen dicke milchige Flüssigkeit, die unter dem Mikroskop zahlreiche Epithelzellen aber auch zahlreiche polynucleare Leucocyten führt.

Die mikroskopische Untersuchung ergab:

Epithel: Das Epithel stellt sich sowohl in der Urethra als über dem Caput gallinaginis als proliferirendes Cylinderepithel dar, indem auf eine oberste Schichte lockerer, theilweise in Abstossung begriffener Cylinderzellen eine auf 3-4 Schichten verbreiterte Lage polygonaler Ersatzzellen folgt.

Subepitheliales Bindegewebe. Dieses erscheint in seinen obersten lockeren Bindegewebsschichten von einem ziemlich dichten Infiltrate durchsetzt, das hauptsächlich aus mononuclearen Rundzellen, wenigen epitheloiden Zellen besteht. Dieses Infiltrat, das nur in den obersten 
Schichten des subepithelialen Bindegewebes sitzt, hört nach unten ziemlich brüske auf.

Drüsen. a) Sowohl der Utriculus als die Drüsen des Caput gallinaginis zeigen Proliferation und Desquamation des Epithels, die bis zu völliger Erfüllung des Lumen mit regellos dicht gedrängt liegenden Epithelzellen führt. Dabei zeigt die Wand des Utriculus, als Fortsetzung des oben beschriebenen subepithelialen Infiltrates, Infiltration der innersten subepithelialen Schichten mit ziemlich dicht stehenden mononuclearen Rundzellen und epitheloiden Zellen.

b) Die Drüsen der Prostata erscheinen zum Theil normal. Ein anderer Theil derselben zeigt ziemlich dichte, aus mononuclearen Rundzellen bestehende Infiltration der Wand. In diesem Infiltrate zahlreiche spindelförmige Anhäufungen scholigen Pigmentes, als Reste alter Hämorrhagien. Im Lumen der periglandulär infiltrirten Drüsen theils nur dicht gedrängte proliferirte Epithelzellen, theils entwed'r anschliesslich, oder neben Epithelzellen auch zablreiche polynucleare Eiterzellen.

Resumé: Chronische entzündliche Infiltration der obersten Schichten des subepithelialen Bindegewebes. Desquamativer, desquamativeitriger Catarrh zahlreicher Prostatadrüsen und des Utriculus bei zum Theil unverändertem periglandulärem Bindegewebe, zum Theil in diesem ein chronisch entzündliches von Hämorrhagien durchsetztes Infiltrat.

Fall VII. (XI.) M. J., 42 J. alt, Magazinarbeiter, starb am 19. Mai 1890 an Tuberculose.

Die Section ergab in den binteren Theilen der Pars pendula bis zum Bulbus sich erstreckend, an der unteren Wand der Harnröhre, im vorderen Theile fast die ganze Circumferenz der Schleimbaut einnehmend, nach Rückwärts sich zungenförmig verschmälernd, eine unregelmässig contourirte Stelle, an der die Schleimhaut glatt, weisslich, zum Theile wie narbig aussieht, während vor, um urd hinter dieser Stelle die Schleimhaut aufgelockert, drusig uneben, mattirt erscheint.

Das Caput gallinaginis etwas vergrössert, wie aus mehreren kleinen Höckerchen bestehend, die Schleimhaut desselben und der Pars prostatica derb, weisslich, zum Theil wie narbig.

Die mikroskopische Untersuchung zeigte:

In der Pars anterior:

Das subepitheliale Bindegewebe, zunächst wuchernd und höckerige Excrescenzen bildend, übergeht durch Bildung derben schrumpfenden Bindegewebes in eine Schwiele, die auch den oberen Theil des Corpus cavernosum in Mitleidemschaft zieht. Das Epithel, anfangs als Cylinderepithel erhalten, proliferirend, übergeht über der Schwiele in flaches, wenig geschichtetes. Plattenepithel und bildet hinter derselben eine aus vielen Lagen von Plattenepithelien bestehende, lockerem Bindegewebe aufsitzende Epithelverdickung. Die Drüsen in der Schwiele bis auf ihre theilweise cystisch degenerirten Ausführungsgänge zerstört, haben an dem Process sonst keinen Antheil. (Details siehe I. Theil, Fall XI.) 
In der Pars posterior:

Epithel: a) der Urethra. Dasselbe stellt sich in ganzer Ausdehnung der Pars prostatica als ein niederes, aus wenigen Schichten bestehendes Plattenepithel dar.

b) Auch das Epithel des Caput gallinaginis ist, soweit erhalten, ein niederes, mehrschichtiges Plattenepithel.

Subepitheliales Bindegewebe: a) Urethra: Dasselbe ist in den obersten Lagen unmittelbar unter dem Epithel in der ganzen Ausdehnung der Pars prostatica in ein derbes, straffes schwieliges Bindegewebe umgewandelt, das viel spindelförmige Kerne führt, wenig Blutgefässe aufweist. Die obersten Schichten dieses schwieligen Bindegewebes sind von einem aus mononuclearen Rundzellen bestehenden Infiltrate durchsetzt, das der Hauptsache nach schütter vertheilt, diffus ist, sich aber an manchen Stellen zu miliaren ruadlichen Knötchen gruppirt, die das Epithel buckelförmig vortreiben. Die tieferen Lagen des subepithelialen Bindegewebes sind normal, locker, wellig.

b) Caput gallinaginis: Gleichwie in der ganzen Pars prostatica, so stellen sich auch im Caput gallinaginis die obersten Schichten des subepithelialen Bindegewebes als derbes, schwieliges, an Zügen von Spindelzellen reiches, straffes Bindegewebe dar, während die tieferen Schichten des subepithelialen Bindegewebes nur leicht infiltrirt sind. Die oberen Schichten des schwieligen Bindegewebes zeigen aber einmal eine recht gleichmässig vertheilte Infiltration mit mononuclearen Rundzellen, dann aber an ganz umschriebenen, sich makroskopisch als kleine Höckerchen kenntlich machenden Stellen, eine ziemlich dichte Durchsetzung der hier seines Epithels beraubten subepithelialen Bindegewebes mit polynuclearen Leucocyten. Die Kerne der Eiterkörperchen sind meist im Zerfall, während anch die obersten Schichten des subepithelialen. Bindegewebes an miliaren umschriebenen Stellen Necrose zeigen.

Drüsen. a) Der Urethra. Diese sowie die Lacunen sind in dem schwieligen subepithelialen Bindegewebe untergegangen.

b) Caput gallinaginis. Die oberfiächlichsten Drüsen des Caput gallinaginis sind in das oben beschriebene Infiltrat eingebettet, comprimirt, ihr Lumen mit abgestossenen Epithelzellen erfüllt. Die tieferen Drüsen des Caput gallinaginis, an deren Wand sich das oben erwähnte, aus mononuclearen Zellen bestehende Infiltrat theilweise herabzieht, sind zum Theil normal, zum Theil zeigen sie Proliferation und Abstossung des Epithels, das im Verein mit zahlreichen Amyloiden das Lumen füllt, zum Theil endlich erscheint das Lumen mancher Drüsen auffällig cystenartig erweitert (wohl durch Compression des Ausführungsganges) und ist dann mit Conglomeraten von Amyloiden und Epithelzellen erfüllt.

c) Prostata. Die Drüsen derselben sind normal.

Resumé: Chronische Entzündung der obersten Lagen des subepithelialen Bindegewebes von Urethra und Caput gallinaginis mit Ausgang in Schwielenbildung und Umwandlung des Epithels in Plattenepithel. Theils diffuse, theils knötchenförmig auftretende Nachschübe subacuter 
Entzündung - Infiltration mit mononuclearen Rundzellen - im schwie. ligen Bindegewebe der Urethra. Fleckweise sehr acut entzündliche Infiltration des schwieligen Bindegewebes - Infiltration mit polynuclearen Lencocyten und Gewebsnecrose - am Caput gallinaginis. Zerstörung der oberfüchlichen Lacunen und Drüsen. Desquamativer Catarrh mit Cystenbildung der Drüsen des Caput gallinaginis.

Fall VIII. (XII.) W. A. 52 Jahre alt, Bahnwächter, starb am 23. April 1890 an Pneumonie.

Die Section ergab in der Pars pendula und im Bulbus die Schleimhaut aufgelockert, oder drusig uneben, dazwischen strahlige oder der Längsrichtung der Urethra quer verlaufende, doch nirgends deutlich stricturirende, deprimirte weissliche Narben.

Die Schleimhaut der Pars prostatica leicht aufgelockert, das Caput gallinaginis vergrössert. Aus der Prostata ein dickes milchweisses Secret auszudrücken, das viel Epithelien und reichlich Eiterzellen, aber keine Amyloide führt.

Die mikroskopische Untersuchung ergab:

In der Pars anterior:

Derbe, das subepitheliale Bindegewebe und die obersten Schichten des Corpus cavernosum durchsetzende, aus chronischer Entzündung desselben (mit Bildung von Höckern) hervorgegangene Schwielen, Umwandlung des Epithels in Plattenepithel, interstitielle und periglanduläre schrumpfende Infiltration der Littre'schen Drüsen. Spuren acuter Entzündung. (Details siebe I. Theil, Fall XII.)

In der Pars posterior.

Epithel: a) der Urethra: Das Epithel der Urethra zeigt sich allüberall als Cylinderepithel mit oberster meist in Abstossung begriffener Schichte von Cylinderzellen und einer auf vier und mehr Reihen verbreiterten Schichte von Ersatzzellen.

b) Am Caput gallinaginis erscheint dem gegenüber das Epithel als mehrschichtiges Plattenepithel, das aus einer unteren Schichte palissadenförmig angeordneter cubischer and mehreren Schichten mittelgrosser polygonaler platter Epithelzellen zusammengesetzt wird.

Subepithel. a) Der Urethra. Das subepitheliale Bindegewebe der Urethra ist locker, wellig und erscheint nur in seinen obersten Schichten von einem lockeren Infiltrate durchsetat, das aus mononuclearen Rundzellen und spärlichen epitheloiden Zellen besteht.

b) Am Caput gallinaginis erscheint das snbepitheliale Bindegewebe im Ganzen derber, straffer, reich an Spindelzellen. Die obersten Lagen desselben sind auch hier von einem nach unten ziemlich scharf absetzenden Infiltrate durchsetzt, das in die Lücken und Spalten des Bindegewebes deponirt, aus mononuclearen Rundzellen, wenigen epitheloiden Zellen, dagegen zahlreichen polynuclearen Leucocyten besteht.

Drüsen. a) Der Urethra. Die Drüsen der Urethra zeigen mit Ausnahme der seichten Lacunen und der ganz oberflächlichen Drüsenbläschen, die beide in dem Infiltrate des subepithelialen Bindegewebes 
sitzen, keine periglandulären Veräßderungen. Dagegen sind sie fast ausnahmslos in der Art verändert, dass ihr Epithel proliferirt, das Lumen fast oder ganz ausfüllt. In zahlieichen Drüsen finden sich aber im Lumen nicht nur proliferirte und desquamirte Epithelzellen, sondern auch bald mehr, bald weniger, polynucleare Leucocyten. Die Menge der Leucocyten überwiegt in manchen Drüsen so sehr, dass ihr Inhalt ein rein eitriger zu sein scheint.

b) Caput gallinaginis. Auch die Drüsen des Caput gallinaginis und der Utriculus zeigen analoge Veränderungen. Keine periglanduläre Errkrankung. Desquamation nnd Proliferation des Drüsenepithels, Erfüllung des Lumen mit desquamirten Epithelien allein, oder mit Epithelien, denen bald mehr, bald weniger, bald sehr viel polynucleare Leucocyten beigemengt sind. Auffallend ist dabei, dass die Drüsen fast gar keine Amyloide führen.

c) Prostata. Die Drüsen der Prostata erscheinen zum Theil normal. Zum Theil ergeben sie den bisher beschriebenen analoge Verhältaisse. Keine periglandulären Veränderungen der Wand, Ausfüllung des Lumen mit desquamirtem Epithel:allein, oder mit Epithel, dem mehr oder weniger polynucleare Leucocyten beigemischt sind, oder der Inhalt scheint nur aus Eiterkörperchen zu bestehen. Auffallend ist auch hier der fast völlige Mangel von Amyloiden.

Resumé. Catarrbalische Desquamation des Cylinderepithels der Urethra, das Epithel des Caput gallinaginis in Plattenepithel verwandelt. Oberflächliche, chronisch-entzündliche Infiltration des subepithelialen Bindegewebes der Urethra und des Caput gallinaginis, am letzteren mit Zeichen acuter Entzündung (polynucleare Leucocyten), theils ein desquamativer, theils desquamativ-eitriger, theils rein eitriger Catarrh der Drüsen der Urethra, des Caput gallinaginis und zahlreicher prostatischer Drüsen, ohne periglanduläre Wanderkrankung.

Fall IX. (XXII.) A. A., 42 J. alt, Tischler, starb am 7. Jan. 1890.

Die Section zeigte in der Mitte der Pars pendula eine strahlige, oberflächliche, deprimirte Narbe, die Schleimhaut um dieselbe weisslich glänzend, verdickt.

Das Caput gallinaginis vergrössert, an dessen linker Seite eine neben der Mündung des Utriculus beginnende, sich nach abwärts ziehende rundliche Narbe.

Die Prostata nicht vergrössert, der linke Ductus ejaculatorius in einen fast rabenfederkieldicken, am Durchschnitte ovalen Gang mit schleimig zähem Inhalt (Epithelien und Lymphkörperchen) umgewandelt.

Bei Druck auf die Prostata entleert sich aus den prostatischen Gängen ein Tropfen dickes, milchweisses Secret, das unter dem Mikroskop neben Amyloiden, zahlreichen eubischen und polygonalen Epithelzellen auch Eiterkörperchen hält.

Die mikroskopische Untersuchung ergab:

In der Pars anterior: chronische, am Wege zur Bildung schwieligen Bindegewebes weit vorgeschrittene Entzündung des subepithelialen 
Bindegewebes mit consecutiver Zerstörung der Lacunen und subepithelialen Drüsen. (Details siehe I. Theil Fall XXII.)

In der Pars posterior:

Epithel: a) Ueber der Urethra ein Cylinderepithel, dessen oberste Schichte der Cylinderzellen vielfach zerworfen, in Abstossung begriffen ist, während unter derselben eine auf $3-5$ Zelllagen verbreitete Schichte von Uebergangszellen folgt. Sowohl zwischen den Cylinder-, als zwischen den Uebergangszellen polynucleare Eiterkörperchen in spärlicher Menge.

b) Ueber dem Caput gallinaginis erseheint das Epithel ausnahmslos, auch über der Narbe als ein mehrschichtiges Plattenepithel, das aus einer untersten Schichte palissadenförmig angeordneter cubischer und darüber mehreren Lagen grosser polygonaler Plattenepithelzellen besteht. Auch hier finden sich zwischen den Epithelzellen spärliche Letrcocyten.

Das subepitheliale Bindegewebe: a) der Urethra ist in einer von oben nach unten abnehmenden Dichte in den obersten Lagen von mononuclearen Rundzellen und epitheloiden Zellen durchsetzt, dem aber auch Spindelzellen und in spärlicher Zahl auch polynucleare Leucocyten beigemischt erscheiuen.

b) Auch am Caput gallinaginis erscheinen die oberen Schichten des subepithelialen Bindegewebes, das sonst locker und wellig ist, von einem aus epitheloiden und mononuclearen Zellen gebildeten Infiltrate durchsetzt.

An einer scharf umschriebenen Stelle jedoch, die der makroskopisch sichtwaren Narbe entspricht und nach links vom Utriculus sitzend, die Ausmündung des linken Dactus ejaculatorius einnimmt, ist das subepitheliale Bindegewebe in eine derbe, aus verflztem, spindelförmige lange Kerne führendem Bindegewebe bestehende Narbe umgewandelt. Innerhalb derselben, sowie insbesondere an deren peripheren, strahlenförmig in das normale Bindegewebe übergehenden Ausläufern Reste von Drüsengewebe eingebettet.

In schnitten unmittelbar hinter der Narbe, sowie in allen hinter der Narbe gelegenen Abschnitten des Caput gallinaginis und der Prostata der linke Ductus ejaculatorius sowohl in seinem aufsteigenden Theil innerhalb des Caput gallinaginis, als während seines horizontalen Verlaufes durch die Prostata sehr bedeutend erweitert, das Epithel desselben, ein niederes Cylinderepithel, unverändert, im Inhalt Epithelien, Pigmentschollen, spärliche Leucocyten und Detritus.

Drüsen: a) Der Urethra. Die spärlichen Lacunen und oberflächlichen Drüsen der Urethra erscheinen in das oben erwähnte subepitheliale Infiltrat eingebettet, ihr Lumen theils mit proliferirendem Cylinder- theils mit reichlichem polygonalen Plattenepithel dicht erfüllt.

b) Caput gallinaginis. Die Drüsen des Caput gallinaginis sind ausnahmslos verändert. Die sehr oberflächlich gelagerten Drüsen erscheinen in das, die obersten Schichten des subepitheliaren Bindegewebes durehsetzende Infiltrat mononuclearer und epitheloider Zellen eingebettet. In der Regel ist auch das Infiltrat um die Drüsen etwas dichter, dringt 
auch etwas tiefer ein. An zahlreichen dieser Drüsen ist durch die Infiltration insbesondere der in das Lumen vorragenden Zotten eine bedeuteude Verengerung des Lumens bedingt, das niedere Cylinderepithel, das diese Zotten deckt, ist oft vollkommen palissadenförmig angeordnet erhalten, das Lumen hält oft dasselbe völlig ausfüllende Amyloide, oft aber wird das Lumen, ausser von Amyloiden auch von zahlreichen, offenbar von Proliferation und Abstossung des Drüsenepithels herrührenden cylindrischen oder polygonalen Epithelzellen erfüllt. Zahlreiche andere Drüsen führen, neben Amyloidkörperchen, proliferirenden, abgestossenen Epithelzellen avch polynucleare Leucocyten in grösserer oder geringerer Zahl.

Analoge Veränderungen zeigt auch der Utriculus, der nur in seinem cberen, der Schleimhautoberfläche benachbarten Antheil periglandulär infiltrirt, dessen buchtige Acini theilweise von normalem, niederen Cylinderepithel ausgekleidet, theilweise aber von proliferirtem Drüsenepithel, polynuclearen Leucocyten bald mehr bald weniger dicht erfüllt sind.

c) Prostata. Die Drüsen der Prostata zeigen ausnahmslos keine periglandulären Veränderungen. Fin Theil derselben erscheint überhaupt normal. Andere dagegen und zwar meist die mehr oberflächlich gelegenen zeigen neben Amyloiden, oft. complete Ausfüllung des Lumens entweder durch proliferirte Epithelzellen allein oder durch proliferirtes Epithel, dem bald mehr, bald weniger polynucleare Leucocyten beigemengt sind. In manchen Drüsen wiegt die Zahl der Eiterzellen so bedeutend vor, dass der Inhalt rein eitrig erscheint, jedoch auch diese Drüsen zeigen weder am periglandulären Bindegewebe noch an dem der Septa und Zotten eine auffallendere Infiitration.

Resumé: Proliferation and Desquamation des noch als Cylinderepithel erhaltenen Epithels der Urethra posterior. Das Epithel des Caput gallinaginis in vielschichtiges Plattenepithel umgewandelt. Oberflächliche subepitheliale, aus mononuclearen Rundzellen und epitheloiden Zellen bestehende Infiltration der Schleimhaut der Urethra und des Caput gallinaginis.

Catarrhalische Desquamation und Proliferation des Epithels der Drüsen der Urethra.

Desquamativer und desquamativ-eitriger Catarrh zahlreicher Drüsen des Caput gallinaginis und der Prostata ohne periglanduläre Erkrankung des Bindegewebes.

Narbige Obliteration des Ausführungsganges des linken Ductus ejaculatorius mit consecutiver bedeutender Erweiterung desselben.

Fall X. (VII.) S. A., 50 Jahre alt, starb am 16. Juni 1890 an Emphysem.

Die Section ergab in der rückwärtigen Hälfte der Pars pendula und im Bulbus die Schleimhaut uneben, aufgelockert, jhr Epithel getrübt, die Follikelöffnungen stecknadelstichgross, klaffend, ihr Rand elevirt.

In der Pars prostatica das Epithel der Urethra und des Caput gallinaginis weisslich träb. Das Caput gallinaginis durch eine in der Gegend der Ausmündung des Utriculus querüber verlaufende schmale 
deprimirte Narbe in zwei Hügelchen mit dazwischen liegenden sattelförmiger Vertiefung getheilt.

Die mikroskopische Untersuchung ergab:

In der Pars anterior: Das Epithel der Schleimhaut und der Lacunen in vieIschichtiges horniges Plattenepithel umgewandelt. Leichte Infiltration der obersten Lagen des subepithelialen Bindegewebes, die nur um die Lacunen und Mündung der Ausführungsgänge der Littre'schen Drüsen zu Knötchen anschwillt, die Littre'schen Drüsen und ihre Gänge als lockeres Infiltrat einschliesst, diesen entlang also in das Corpas cavernosum eindringt, sonst aber oberflächlich bleibt, aus mononuclearen und epitheloiden Zellen besteht. Kolossale von den Ersatzzellen der Drüsenausführungsgänge ausgehende, mit Umwandlung in Plattenepithel einhergehende Wucherung der Auskleidung der Ausführungsgänge der Littre'schen Drüsen, die sich nicht nur im Gange zwischen die innersten Schichten der noch erhaltenen Cylinderepithelien und die Basalmembran, sondern auch in den Drüsenkörper zwischen das secernirende Epithel der Acine und deren periglanduläres Bindegewebe einschiebt. (Details, siehe I. Theil Fall VII.)

In der Pars posterior:

Epithel: a) Der Urethra: Das Epithel der Urethra ist ein proliferirendes Cylinderepithel, eine oberste Schichte theils noch schön palissadenförmig angeordneter, theils und hauptsächlich aber zerworfener, in ihrem Zusammenhange gelockerter, theilweise sich ablösender Cylinderzellen, die eiver bis zu fünf und mehr Lagen verbreiterten Schichte polygonaler Frsatzzellen aufruhen.

b) Ueber dem Caput gallinaginis, in dessen ganzer Ausdehnung ist das Epithel ein vielschichtiges Plattenepithel, das aus einer untersten Lage cubischer und zahlreichen Lagen polygonaler auffällig grosser Epithelzellen besteht.

Subepitheliales Bindegewebe: a) Der Urethra: Das sub. epitheliale Bindegewebe der Urethra, das zart, locker und wellig erscheint, ist in seinen obersten Lagen von einem dichten Infiltrate durchsetzt, das aus mononuclearen Rundzellen und epitheloiden Zellen, zahlreichen hyalinen Kugeln, besteht und nach unten zu ziemlich unvermittelt, brïsk abbricht.

Dieses Infiltrat, im Ganzen schon dicht, ist um die seichten Lacunen der Urethra noch dichter angehäuft und zeigt ausserdem noch an manchen Stellen besonders dichte, knötchenförmige Anhäufungen.

b) Am Caput gallinaginis. Auch hier erscheint das subepitheliale Bindegewebe von einem dem oben beschriebenen analogen Infiltrate in semen obersten Schichten durchsetzt, ist sonst locker und wellig.

Nur an einer Reihe aufeinanderfolgender Schnitte, die der oben beschriebenen Einsenkung des Caput gallinaginis entsprechen, erscheint das subepitheliale Bindegewebe in anderer Weise verändert. Es ist hier von keinem Infiltrate durchsetzt, im Gegentheil zellarm, aber straff, derb, von langen spindelförmigen Kernen durchzogen, verfilzt, also narbig. 
Dieser Narbenzug steigt in dem Winkel zwischen Urethra und Caput gallinaginis auf, zieht sich als ein schmales derbes Band unmittelbar unter dem Epithel über die ganze Wölbung des Caput gallinaginis und reicht auf der anderen Seite wieder bis zum Winkel zwischen Urethra und Caput gallinaginis. Beiderseits übergeht dieses narbige Band allmälig in das normale Bindegewebe und das Faserwert der Prostata.

In diesem Narbengewebe fehlt jede Andeutung von Drüsengewebe, ebenso sind die bis an die Narbe zu verfolgenden Ductus ejaculatorii und der Utriculus durch dieses verschlossen.

Drüsen: a) Der Urethra. Die seichten Lacunen und spärlichen oberflächlichen Drüsen der Schleimhaut der Urethra sind in das oben beschriebene subepitheliale Infiltrat eingebettet, ihr Epithel proliferirt und füllt meist das Drüsenlumen völlig aus.

b) Des Caput gallinaginis. Von den Drüsen des Caput gallinaginis nimmt zunächst der Utriculus unsere Aufmerksamkeit in Anspruch. Derselbe, ist, wie oben erwähnt, hart an seiner Ausmündung narbig verschlossen. Sein Lumen erscheint auffällig erweitert und vergrössert, so dass derselbe nicht nur innerhalb des Caput gallinaginis, sondern auch tief im Gewebe der Prostata, zwischen den beiden Ductus ejaculatorii nachzuweisen ist. Die de norma zahlreichen Buchten und Nischen sind durch die Erweiterung ausgeglichen, so dass das Lumen am Durchschnitt sich als ein gleichförmiges Oval mit seicht buchtigen, vielfach concaven Contouren präsentirt. Die Wand desselben ist in einer von oben nach unten abnehmenden Intensität, im Ganzen aber nur sehr mässig und nur in dünner subepithelialer Lage von einem lockeren Infiltrat mononuclearer Rundzellen durchsetzt. Das Epithel präsentirt sich als proliferirendes Cylinderepithel, eine oberste Schichte sehr niederer palissadenförmig festgefügter Cylınderepithelien mit scharf hervortretendem Kerne, unter der an verschiedenen Stellen wechselnd zwei, drei, fünf Lagen kleiner polygonaler Zellen liegen. Das Lumen ist dicht angefüllt mit einem aus polygonalen Zellen, Pigmentschollen, Detritus gebildeten Inhalt.

Von den übrigen Dräsen des Caput gallinaginis sind die vor und hinter der Narbe gelegenen obertächlichen in das obertächliche subepitheliale Infiltrat eingebettet, ihr Lumen von proliferirendem Epithel und grösseren und kleineren Amyloiden erfüllt.

Unterhalb der Narbe ist eine grössere Zahl dieser Drüschen durch Verschliessung ihrer Mündung in kleine Cystchen umgewandelt, deren Wand von einem niederen Cylinderepithel ausgekleidet ist, deren Inhalt, soweit nicht beim Schneiden ausgefallen, aus grossen Amyloiden and Epithelzellen besteht, während andere dieser Cystchen völlig mit dicht gedrängten festgefügten polygonalen Zellen erfüllt erscheinen.

c) Prostata. In der Prostata sind es zunächst die Ductus ejaeulatorii, deren Veränderungen uns auffallen. An ihrer Ausmündung durch Narbengewebe verschlossen, sind beide Ductus ejaculatorii in ihrem Lumen bedeutend $\epsilon$ rweitert. Diese Erweiterung ist im ganzen Verlauf sowohl innerhalb des Caput gallinaginis, als in der Prostata nachzuweisen. 
Trotz der Erweiterung sind aber die vielfachen Nischen und Buchten der Wand nicht ausgeglichen, so dass das Lumen einen ungemein vielzackigen, buchtigen Contour darbietet.

Die Wand der Ductus ejaculatorii ist nur locker infiltrirt, die Blutgefässe derselben sehr erweitert. An zahlreichen Stellen ältere und frischere Hämorrhagien unter das Epithel, die dasselbe oft in recht weiter Ausdehnung abheben. An einigen Stellen, durch Zerreissung des Epithels, directe Blutung in das Lumen. Das Epithel präsentirt sich als festgefügtes Cylinderepithel mit einer oder zwei Schichten Uebergangszellen. Im Lumen Epithelzellen, Detritus, Pigmentschollen, rothe Blutkörperchen in grosser Menge.

Die Drüsen der Prostata zeigen ausser partieller Erfüllung ihres Lumens mit proliferirtem Epithel, Amyloiden nichts Abnormes, deren Wand unverändert.

Res u $\mathrm{m}$ é. Catarrhalische Desquamation und Proliferation des noch als Cylinderopithel erhaltenen Epithels der Urethra posterior. Das Epithel an Caput gallinaginis in vielschichtiges Plattenepithel umgewandelt.

Oberfächliche subepitheliale, dichte, aus mononuclearen Rundzellen und epitineloiden Zellen bestehende Infiltration der Schleimhaut von Urethra und Caput gallinaginis mit dichterer Anhäufung um die oberflächlichen Drüsen und stellenweise Gruppirung zu Knötchen.

Umwandlung des subepithelialen Bindegewebes am Caput gallinaginis an einer scharf begrenzten Stelle in eine lineare, quere, die Mündung von Caput gallinaginis, Ductus ejaculatorii und einigen prostatischen Drüsen verschliessende Narbe.

Catarrhalische Desquamation und Proliferation des Epithels der Drüsen der Urethra. Rein desquamativer Catarrh zahlreicher Drüsen des Caput gallinaginis und der Prostata ohne periglanduläre Veränderungen.

Obliteration der Mündung der Ductus ejaculatorii und des Utriculas mit consecutiver Erweiterung von deren Lumen, Proliferation des Epithels, Infiltration der Wand. In den Ductus ejaculatorii capillare subepitheliale sowie Hämorrhagien in das Lumen.

Fall XI. (XXIII.) P. A., Diener, 53 Jahre alt, starb am 20. Jänner 1890 an Pneumonie.

Die Section zeigte die Pars pendula urethrae 5 Ctr. hinter dem Orificium beginnend bis nahe an den Bulbus von zahlreichen theils strahligen, theils quer verlaufenden, nicht auffallend stricturirenden Narbensträngen durchzogen.

In der Pars prostatica die Schleimhaut narbig glänzend, bis an das Ostium vesicae von zarten, queren narbigen Leisten durchzogen.

Das Caput gallinaginis vergrössert, durch eine quere, die Ductus ejaculatorii und die Mündung des Utriculus verschliessende, deprimirte lineare schmale Narbe in zwei Höcker getheilt.

Die Prostata etwas vergrössert, bei Durchschnitt von unzähligen, is kleinen Höhlen gelegenen braunen, harten Concretionen durchsetzt. 
Die mikroskopische Untersuchung ergab in der Pars anterior: chronische Entzündung des subepithelialen Bindegewebes mit dem Ausgang in Schrumpfong und consecutive Zerstörung der Lacunen und hochgelegenen Schleimdrüsen. (Siehe I. Theil, Fall XXIII.)

Eine microscopische Untersuchung der Pars posterior konnte leider nicht vorgenommen werden, da die zahllosen, durch die ganze Prostata und Caput gallinaginis verstreuten Concretionen eine Zerlegung des Gewebes mit dem Mikrotom absolut unmöglich machten.

Ueberblicken wir nun die soeben mitgetheilten Fälle, indem wir uns zunächst dem makroskopischen Bilde zuwenden.

Dieses ist naturgemäss ein lückenhaftes. Jene feinen, auf Hyperämie, seröser Durchtränkung etc. beruhenden Veränderungen, die das Endoskop in vivo finden lässt, sind an der Leiche natürlich erkenntlich.

Nichtsdestoweniger bietet die Schleimhaut der Urethra sowohl, als das Caput gallinaginis deutliche und marcante Veränderungen.

Die Schleimhaut der Urethra innerhalb der Pars prostatica erscheint zunächst aufgelockert. Diese Lockerung ist nur zart, kaum angedeutet, gibt der Oberfäche ein mattirtes, gesticheltes Aussehen. (Fall I.) Die Auflockerung kann aber bedeutend sein und in einigen Fällen (Fall III-V, VIII) notirten wir und wiesen auch mikroskopisch ein papillares, zottiges Auswachsen der Schleimhaut der Urethra nach.

Meist ist diese papillare Beschaffenheit der Schleimhaut um das Caput gallinaginis am meisten ausgesprochen, ja sie geht seitlich auch auf das Caput gallinaginis hinauf, wo sie aber successiv abnehmend sich verliert. Ebenso wird diese Veränderung auch nach rückwärts zu immer weniger accentuirt, bis sie am Ostium vesicae oder frïher völlig aufhört, nie aber über dieses hinaus in die Blase sich erstreckt.

Im Gegensatz zu diesen Fällen, in denen eine Auflockerung der Schleimhaut nachweisbar ist, ist in einigen anderen Fällen das Gegentheil, eine Verdickung, schwielige Degeneration, Bildung derben Bindegewebes zu constatiren. (Fall VII, IX-XI.) In einem Fall (XI) ist sogar der Befund leistenförmiger Narben in der Pars posterior notirt. Mikroskopische Untersuchung gerade dieses Falles war nicht möglich, so dass es dahin ge- 
stellt bleiben muss, $a b$ es sich hier um wirkliche leistenförmige Narben, oder nur um die im ersten Theile bereits genau beschriebenen leistenförmigen Epithelverdickungen und Bildungen von Plattenepithel handelt, die in der Urethra anterior sowohl für den Anatomen als für den Endoskopiker so leicht Narben vortäuschen.

Meine besondere Aufmerksamkeit wandte ich immer dem Caput gallinaginis zu. In der That zeigt dieses auch interessante Veränderungen. Der schleimbäutige Ueberzug desselben ist einmal aufgelockert, wie gestichelt (Fall I). Die für die Harnröhrenschleimbaut bereits beschriebene papillare Auflockerung der Schleimhaut kann auch auf den schleimhäutigen Ueberzug, aber nur die Seitentheile des Caput gallinaginis übergreifen. (Fall V.)

In Gegensatz dazu erscheint der schleimhäutige Ueberzug des Caput gallinaginis in anderen Fällen mehr straff, schwielig. Diese schwielige Beschaffenheit ist auf eine circumscripte Stelle an der Kuppe beschränkt (Fall II) oder betrifft die Schleimhaut in toto (Fall VII-XI).

Aber nicht nur das Aussehen, auch Grösse und Form des Caput gallinaginis zeigt Veränderungen.

Was ersteres betrifft, so ist meist eine Vergrösserung des Caput gallinaginis notirt. In anderen Fällen ist es normal gross, eine auffällige Verkleinerung fand ich nicht.

Interessant sind die Veränderungen der Form. So ist in einem Falle (VII) das Caput gallinaginis nicht ein einziger glatter Höcker, sondern es besteht aus mebreren seichten Höckerchen mit dazwischen ziehenden seichten Depressionen.

Interessant und pathologisch bedeutsam sind die Narbenrespective Schwielenbildungen am Caput gallinaginis.

So sitzt (Fall IX) an der linken Seite des Caput gallinaginis eine circumscripte Narbe, die den linken Ductus ejaculatorius verschliesst.

In zwei Fällen (Fall $\mathrm{X}$ und $\mathrm{XI}$ ) ist das Caput gallinaginis durch eine schmale, querverlaufende Narbe in zwei Höcker, einen vorderen und einen hinteren, die durch eine jochförmige Einsenkung mit einander verbunden sind, getheilt. In beiden 
Fällen verschliesst diese schmale Schwiele beide Ductus ejaculatorii und die Mündung des Utriculus.

Weiters wurde von mir das Prostatasecret, dessen Menge und Beschaffenheit ins Auge gefasst. Ich gewann dasselbe, indem ich, nachdem die Urethra von oben durchschnitten und ausgebreitet war, auf das Gewebe der Prostata drückte und das am und um das Caput gallinaginis aus den Ductus prostatici austretende Secret betrachtete, mikroskopisch untersuchte. In einer Reihe von Fällen erschien das dünnflüssig, milchige Secret makroskopisch und mikroskopisch normal.

In anderen Fällen dagegen fiel das Secret schon dem blossen Auge durch seine dickere Consistenz, seine sattweise Farbe auf.

Mikroskopische Untersuchung gestattete diese Fälle in zwei Gruppen zu sondern.

In der einen Gruppe (Fall I, III) hält das Prostatasecret, mikroskopisch untersucht, auffällig viel Epithelzellen, wobei die Menge der Amyloide schwankt. Die Epithelzellen haben bald mehr den Charakter von Cylinderzellen, bald ist es schwer, sich zwischen niederem Cylinderepithel oder cubischem Epithel zu entscheiden, bald sind sie polygonal.

In einer zweiten Gruppe von Fällen (Fall VI, VIII, IX) finden wir im Prostatasecret neben den oben beschriebenen Epithelzellen, Cylinder- oder cubischen Epithelzellen, polygonalen Zellen reichlich polynucleare Leucocyten. Die Amyloide sind, was Menge und Constanz betrifft, auch hier unverlässlich, werden wir übrigens über dieselben noch sprechen.

Wenden wir uns nun jenen Veränderungen zu, die die einzelnen am anatomischen Bau der Urethra und Prostata betheiligten Gebilde bei mikroskopischer Untersuchung erkennen lassen.

\section{A. Epithel.}

a) Epithel der Harnröhre. Das Epithel der Harnröhre zeigt Veränderungen, die den in der Pars anterior beschriebenen völlig analog sind.

De norma ein Cylinderepithel, das aus einer oberen Reihe palissadenförmig angeordneter festgefügter Cylinderzellen, einer 
oder zwei unterliegenden Reihen polygonaler Ersatzzellen besteht, nimmt es an der Erkrankung zunächst in einer Reihe von Fällen (Fall I-VI und VII-X) in der Art theil, dass es zu einer Desquamation, Proliferation, schleimigen Degeneration kommt. Die Reihe der Cylinderzellen verliert ihren Zusammenhang, die Zellen sind gelockert, zerworfen, einzelne oder Gruppen sind abgestossen. Dabei erscheinen die einzelnen Cylinderzellen oft gequollen, schleimig degenerirt. Die untere Schichte der polygonalen Ersatzzellen pflegt dann durch Proliferation, Vermehrung derselben statt der gewöhnlich ein bis zwei Reihen auf mehrere, vier, sechs und mehr Reihen verbreitert zu sein. Ist die Verbreiterung eine bedeutende, so pflegt dann meist die unterste Reihe nicht aus polygonalen, sondern aus niederen cubischen Zellen zu bestehen.

Eine uns wohlbekannte Veränderung ist der Uebergang des Cylinderepithels in Plattenepithel. Dieses Plattenepithel kann, wie aus dem ersten Theile der Arbeit, aus den Studien an der Pars anterior ersichtlich ist, nach drei Typen gebaut sein.

1. Auf eine Schichte polygonaler Ersatzzellen, die verbreitert und auf sechs und mehr Lagen angewachsen ist, folgt eine Lage niederer platter Epithelzellen (Typus der Schleimhäute mit Plattenepithel).

2. Das Epithel besteht aus einer unteren Schichte cubischer Zellen, auf die dann zahlreiche Lagen grosser polygonaler, nach oben zu immer grösser werdender Zellen folgte, die an manchen Stellen selbst als Riffzellen erscheinen. (Epithel nach dem Typus des Rete Malpighi.)

3. Das Epithel besteht aus wenigen Lagen niederer, platter, am Durchschnitt spindelförmiger Zellen. (Typus des Epithels über Narben.)

Von diesen drei in der Urethra anterior zu constatirenden Typen fand ich in der Urethra posterior nur in einem Falle (Fall VII) ein Analogon. Und zwar war das Epithel in diesem Falle nach dem dritten Typus gebaut, wobei zu bemerken, dass ganz analog zu den Beobachtungen in der Urethra anterior es sich auch hier um schwielige Degeneration des subepithelialen Bindegewebes handelte. 
b) Epithel des Caput gallinaginis. Die Veränderungen desselben zeigten sich vielgestaltig, wobei ich sofort hervorheben will, dass das Epithel des Caput galli$\mathrm{nag}$ in is ein de norma niederes Cylinderepithel mit ein bis zwei Reihen polygonaler Ersatzzellen, rascher die Tendenz hat in Plattenepithel überzugehen, als das der umgebenden Urethra.

So sind unter den angeführten Fällen mehrere verzeichnet, wo das Epithel der umgebenden Urethra sich noch als proliferirendes Cylinderepithel darstellt, während am Caput gallinaginis schon Plattenepithel sitzt. Diese Veränderung scheint am Caput gallinaginis von der Kuppe nach den Seiten vorzuschreiten, wenigstens sind zwei Fälle (Fall II und IV) beachtenswerth, in denen an der Kuppe des Caput gallinaginis Pflasterepithel, an den Abhängen noch Cylinderepithel zu constatiren ist.

Die Veränderungen des Epithels sind im Uebrigen die bekannten.

Zunächst einmal ist das Cylinderepithel noch als solches erhalten, aber die Reihe der Cylinderzellen ist gelockert, die Zellen verschoben, Gruppen derselben abgetallen. Dabei sind einige oder zahlreiche Zellen gequollen, schleimig degenerirt. Die Schichte der Ersatzzellen ist unter diesen Cylinderzellen stets auf oft zahlreiche Lagen verbreitert.

Oder aber das Cylinderepithel ist in Plattenepithel umgewandelt, von dem ich am Caput gallinaginis zwei Typen nachweisen konnte: Den früher beschriebenen zweiten Typus, untere Schichte cubischer Zellen, zahlreiche Schichten polygonaler, nach oben zu immer grösser werdender platter Epithelzellen (Fig. II $a$ ) (Typus des Rete Malpighi) ohne Riffzellen und den früher beschriebenen dritten Typus, wenige Lagen niederer, platter, am Durchschnitt spindelförmiger Epithelzellen (Fig. III $a$, Fig. IVa). (Typus des Epithels über Narben.)

Die schon in der Urethra anterior zu constatirende Thatsache: dass die Umwandlung der Cylinder- in Plattenepithel mit den Veränderungen im subepithelialen Gewebe in einem gewissen Zusammenhang steht, liess sich auch hier constatiren. So war über frischerer 
Rundzelleninfiltration das Epithel noch als Cylinderepithel erhalten (Fall I, III, VI), über älterem in bindegewebiger Umwandlung begriffenen Infiltraten fand sich Epithel nach dem zweiten Typus (Fall IV, VIII-X) über altem schwieligen Bindegewebe Epithel nach dem dritten Typus (Fall II, VII). Ja selbst in ein und demselben Falle zeigte sich, dass circumscripte Schwielen (Fall II und IV) von Plattenepithel, jüngere Rundzelleninfiltrate in der Umgebung der Schwiele noch von Cylinderepithel gedeckt waren.

$\mathrm{Zu}$ erwähnen ist noch, dass bei Nachschüben acuter Entzündung, Infiltration mit polynuclearen Leucocyten im subepithelialen Bindegewebe, solche auch zwischen den Epithelzellen nachzuweisen waren.

\section{$B$. Subepitheliales Bindegewebe.}

a) Urethra. Wie in der Pars anterior, so spielt sich auch in der Pars posterior ein wesentlicher Act des ganzen chronischen Entzündungsprocesses in dem subepithelialen Bindegewebe ab. Auch hier haben wir es mit einem chronischen Entziindungsprocesse zu thun, der mit zelliger Infiltration und Proliferation der Bindegewebszellen beginnt und bei dem das Infiltrat die ausgesprochene Tendenz hat, wieder in Bindegewebe überzugehen, das entsprechend seiner entzündlichen Provenienz, meist einen derben, schwieligen Charakter anzunehmen bestrebt ist.

Das subepitheliale Bindegewebe zeigt in unseren Fällen alle diese Uebergänge vom Infiltrat zur Schwiele.

So haben wir zunächst in einer Reihe von Fällen, die wir gewiss als die frischeren ansprechen können, in den oberen Schichten des subepithelialen Bindegewebes ein Infiltrat (Fall I, II, VI, VIII-X), das aus mononuclearen Rundzellen besteht, denen bald mehr, bald weniger epitheloide Zellen beigemengt $\sin$, d. h. Zellen, die sich durch grösseren Drotoplasmareichthum, grosse blass tingible Kerne auszeichnen. Die Fibrillen des Bindegewebes, zwischen die das Infiltrat deponirt ist, sind in diesem Stadium zart, locker und wellig.

Das Infiltrat selbst zeigt nach Intensität und Extensität Varianten. Bald ist dasselbe lockerer, die Zellansammlungen. 
durch sichtbare Züge von Bindegewebe getrennt, bald ist es dichter, ja so dicht, dass innerhalb des Infiltrates von Bindegewebszügen njchts mehr zu sehen ist, die obersten Lagen des subepithelialen Bindegewebes ganz aus neben und über einander gelagerten, dichtgedrängten Rundzellen bestehen. Ebenso wie die Dichte, so variirt aber auch die Tiefe, in die das Infiltrat dringt, sehr bedeutend. Das Infiltrat pflegt dann endlich wieder entweder nach der Tiefe allmälig lockerer zu werden, so dass ein allmäliger Uebergang zum Gesunden geschaffen ist, es kann aber (Fall I, VI) das Infiltrat überall die gleiche Dichte haben und nach unten zu scharf absetzen, unvermittelst ins Gesunde übergehelı.

Den höchsten Grad nach In- und Extensität des Infiltrates stellt wohl Fall I dar. (Fig. Ib.) Hier steht Zelle an Zelle bis in bedeutende Tiefe, wo das Infiltrat brüsk aufluört. Die Intensität der Entzündung documentirt sich aber auch in der Bildung von Heerden inselförmiger Necrose in den obersten Partien des Infiltrates (Fig. Ic), Stellen an denen die Contouren der Zellen noch sichtbar sind, die Kerne derselben aber ihre Tinctionsfähigkeit eingebüsst haben, nicht sichtbar sind.

Als ältere Fälle werden wir jene Fälle betrachten, in denen wir auch noch dasselbe Infiltrat von mononuclearen Rundzellen und epitheloiden Zellen constatiren können, in denen aber zwei weitere Momente hinzukommen. Einmal das Auftreten von spindelförmigen Zellen in dem Infiltrat in grösserer oder geringerer Zahl, dann aber zu constatiren ist, dass die oberen Schichten des subepithelialen Bindegewebes ihren Charakter als lockeres, welliges Bindegewebe verloren haben und sich, im Gegensatz zu den tieferen noch normalen Bindegewebszügen als ein strafferes, derberes, faseriges, an Spindelzellen reiches Bindegewebe darstellen. Wir werden wohl nicht irre gehen, wenn wir annehmen, dass sich in diesem Bindegewebe schon vor einiger Zeit ein chronischer Entzündungsprocess abspielte, der mit seinem Ausgange eben das Bindegewebe derber, straffer machte und dass die nun noch zu constatirende RundzellenInfiltration entweder Residuen des Entzündungsprocesses darstellen oder einem Nachschube desselben chronischen Entzündungsprocesses ihre Entstehung verdankt. Ein solcher Nach- 
schub kanu diffus, er kann aber auch (Fall VII) mehr localisirt sein. Das Bindegewebe ist in toto in seinen obersten Schichten schwielig, die Rundzelleninfiltration tritt aber nicht diffus, sondern mehr knötchenförmig auf.

Eine eigene Gruppe stellen mehrere Fälle dar. (Fall III-V.)

Das Bindegewebe ist in denselben in besonders intensiver Weise an dem Entzündungsprocesse betheiligt. Es wächst aus. Das subepitheliale Bindegewebe verliert dann seinen oberen glatten oder leicht welligen Contour, derselbe wird grob zackig und buchtig, das subepitheliale Bindegewebe ist zu einer Reile von Papillen a usgewachsen, die entweder spitz-conisch oder einfach höckerig oder an der Spitze kolbig angeschwollen sind.

Das Epithel folgt dann diesen Auswïchsen, indem es ihrem Contour nachahmt, die so gebildeten Berge und Thäler als dünnes Band überzieht und contourirt. An diesen papillaren Excrescenzen sind zwei Factoren betheiligt, einmal das infiltrirte und proliferirende Bindegewebe, dann aber meist auch die Blutgefässe. Diese letzteren wohl durch Erweiterung und Neubildung. Wenigstens finden sich (Fall III) in dem subepithelialen Bindegewebe sowohl der Excrescenzen als deren Basis so zahlreiche Blutgefässe, die, obwohl sie den Charakter von Capillaren bewabren, doch ein so grosses Lumen zeigen, oft so hoch unter das Epithel ansteigen, dass sie dem Gewebe grosse Aehnlichkeit mit Schwellgewebe verleihen.

Endlich kann es neben Nachschüben chronischer auch zu Schüben acuter Entzündung, Extravasation und Durchsetzung des Gewebes mit polynuclearen Leucocyten kommeu (Fall IX, Fig. II $b$ ).

b) Caput gallinaginis. Die Veränderungen des subepithelialen Bindegewebes im Caput gallinaginis sind im Grossen und Ganzen den für die Urethra geschilderten analog. Wir können also auch hier, dem Alter des Processes entsprechend, zwei Stadien, das der entzündlichen Infiltration und das der Schwielenbildung unterscheiden. In ersten Stadien finden wir auch hier ein Infiltrat, das aus mononuclearen Rundzellen und epitheloiden Zellen besteht (Fig. II $b$ ), verschiedene Dichte besitzt, bald mehr 
bald weniger tief dringt, nach unten zu allmälig oder mit scharfer Grenze in das gesunde Gewebe übergeht. In den frischeren Fällen ist dieses Infiltrat in ein lockeres, welliges Bindegewebe deponirt. Als interessanter Fall dieser Gruppe gilt auch hier Fall I (Fig. I $a$ ), wo es an der Oberfläche des Infiltrates zu ganz oberflächlicher, inselförmiger Necrose an mehreren Stellen kam (Fig. I $b$ ).

In den älteren Fällen äussert sich die Tendenz zum Uebergang in derbes Bindegewebe durch zwei Momente, einmal dadurch, dass in dem Infiltrate neben Rundzellen und epitheloiden Zellen reichlich Spindelzellen auftreten, dann aber dadurch, dass das Bindegewebe, in welches das Infiltrat deponirt erscheint, straffer, derber wird (Fall III, VI, VII, VIII, Fig. III $b$ ).

In allen Fällen aber spielen sich die eben geschilderten Veränderungen nur in den obersten Schichten des Caput gallinaginis ab, die tieferen Schichten sind in allen Fällen locker, wellig, also normal.

Wenn sich nun auch die Veränderungen des subepithelialen Bindegewebes von Urethra posterior und Caput gallinaginis im Grossen gleichen, so sind doch einige aufällige Differenzen zu notiren.

Zunächst scheint das subepitheliale Bindegewebe des Caput gallinaginis weniger die Tendenz zu haben, in Form papillarer Excrescenzen a u z u w a chsen. Von den drei Fällen, in denen ein Anwachsen des subepithelialen Bindegewebes der Urethra zu papillaren Excrescenzen nachweisbar war (Fall III-V), umgeben in zwei Fällen diese papillaren Excrescenzen das Caput gallinaginis von allen Seiten, reichen nach rückwärts bis an das Ostium vesicae, aber sie ziehen nicht an dem Caput gallinaginis hinauf.

Nur in einem Falle (Fall IV) steigen diese papillaren Excrescenzen auch seitlich an Caput gallinaginis hinauf, um successive immer niederer zu werden und erreichen die Kuppe des Caput gallinaginis nicht.

Diese Thatsache findet ihre Erklärung vielleicht in dem zweiten, nun zu erwähnenden Momente. Vergleichung der Veränderungen in dem subepithelialen Bindegewebe der Urethra und des Caput gallinaginis geben den Eindruck, als wenn 
der chronische Entzündungsprocess am Caputgallinaginis rascher abliefe, rascher in das zweite Stadium der Bindegewebsbildung überginge, als in der benachbarten Urethralschleimhaut. Wenigstens finden wir mehrere Fälle (Fall II-V, VI, VII), in denen das subepitbeliale Infiltrat in der Urethra sich noch vorwiegend im ersten Stadium der Rundzelleninfiltration findet, am Caput gallinaginis dagegen schon weiter am Wege der Bildung schwieligen Bindegewebes rorgeschritten ist.

Unsere besondere Aufmerksamkeit beanspruchen die $\mathrm{Narben-}$ und Schwielenbildungen am Caput gallinaginis.

Hier ist zunächst Fall IX beachtenswerth (Fig. IV b). Eine rundliche, tiefe, mitten in das subepitheliale Gewebe eindringende Narbe hat den Ductus ejaculatorius der linken Seite obliterirt. Die wenn auch mit strahlenförmigen Ausläufern versehene, doch in Totalansicht rundliche Narbe links rom Utriculus, ihr tiefes Eindringen, die Thatsache, dass die linkerseits von der Narbe eingenommene Partie, auf der rechten völlig correspondirenden Seite von mehreren Drüschen eingenommen wird, dass in der Narbe selbst noch Drüsenreste nachweislich, lässt es als wahrscheinlich erscheinen, dass diese Narbe einem folliculären und perifolliculären Drüsenabscesse ihren Ursprung verdankte, der nach der Urethra zu durchbrach, das Endstück des Ductus ejaculatorius in den Bereich der Vereiterung, also dann bei der Vernarbung auch in den Bereich der Narbe zog.

Ebenso interessant sind die oberflächlichen, deprimirten Narben oder, wie ich glaube, in diesen Fällen richtiger Schwielen (Fall II, X, XI). Die eine dieser Schwielen sitzt als circumscripte Delle an der Kuppe des Caput gallinaginis (Fall II). Sie besteht aus derbem, an Spindelzellen reichen Bindegewebe, das von Plattenepithel gedeckt ist.

Die beiden anderen auch aus einem schmaler Bande von derbem, an Spindelzellen reichen Bindegewebe mit Plattenepithel gebildeten Schwielen ziehen im Bogen quer über das Caput gallinaginis hinweg, theilen durch ihre Depression dasselbe in zwei Höcker und obliteriren vermöge ihres Sitzes beide Ductus ejaculatorii und die Mündung des Utriculus. 
Wie die Entstehung dieser bandförmigen Schwielen zu erklären, steht nicht ganz fest. Würde es sich um bindegewebige Schrumpfung des ganzen Caput gallinaginis handeln, so dürfte man dies gewiss einfach auf Schrumpfung des entzündlichen, bindegewebig umgewandelten Infiltrates auffassen. Bei der umschriebenen, einmal runden, dann als queres Band uber die höchste Circumferenz des Caput gallinaginis verlaufenden Form geht das wohl nicht gut an.

Vielleicht aber wirft Fall II auf die Genese dieser Schwielen ein Licht.

Wir erwähnten, dass in diesem Falle ein sehr bedeutendes, tief dringendes, aus dicht gedrängten Rundzellen bestehendes Infiltrat das subepitheliale Bindegewebe von Urethra und Caput gallinaginis durchsetzt und an mehrfachen inselförmigen Stellen eine oberfächliche Necrose des Infiltrates eingetreten ist. Diese Necrose könnte gelegentlich tiefer ins Gewebe eingreifen. Die so necrotisirten Partien würden im weiteren Verlauf abgestossen und durch Narbengewebe ersetzt werden. So würde die circumscripte schwielige Delle in Fall II in einer einzigen isolirten Necrose, die bandförmigen Schwielen in Fall $X$ und XI durch eine bandförmige oder mehrere inselförmige, confluirende Necrosen über die grösste Circumferenz des Caput gallinaginis ihre Erklärung finden.

Wie überall in der Urethra, so kann auch am Caput gallinaginis der chronische Process zu jeder Zeit durch einen Schub acuter Entzündung complicirt werden, wovon Fall VIII (Fig. II $b$ ) und IX schöne Beispiele liefern.

Im Fall VIII sind die obersten Schichten des subepithelialen Bindegewebes straff, derb, von Rundzellen und wenigen epitheloiden Zellen, aber auch von reichlichen polynuclearen Leucocyten durchsetzt. Wir blicken hier in drei Phasen des Entzündungsprocesses gleichzeitig. Als Resultat einer älteren Phase chronischer Entzündung haben wir die schwielige Degeneration des Bindegewebes anzusehen. Eine zweite, frischere Phase desselben Entzündungsprocesses producirt sich nur als Rundzelleninfiltration, während die Infiltration des Gewebes mit polynuclearen Leucocyten uns eine Phase acuter Entzündung anzeigt. 
Besonders interessant ist aber Fall VII, in dem die obersten Schichten des subepithelialen Bindegewebes sich schon als sehr derbes, schwieliges Gewebe produciren, das von einem lockeren Rundzelleninfiltrate durchsetzt ist, während gleichzeitig an mehreren Stellen inselförmige Schübe acuter Entzündung circumscripte Infiltration mit polynuclearen Leucocyten - auftraten, die in Folge ihrer Knötchenform und der Mehrzahl dieser Knötchen dem Caput gallinaginis makroskopisch schon das Aussehen verliehen, als bestele dessen Oberfläche aus mehreren kleinen Knötchen und dazwischen verlaufenden Einsenkungen, also Aehnlichkeit mit einer Himbeere darboten.

\section{G. Drüsen.}

a) Urethra. Die Schleimhaut der Urethra posterior ist an Lacunen und Drüsen arm. Die Lacunen stellen sich meist nur als ganz seichte Einbuchtungen dar, die de norma von Cylinderepithel ausgekleidet sind. Die Drüsen der Urethra, von acinösem Bau, liegen oberflächlich, bestehen aus wenigen Acinis und sehr kurzem Ausführungsgang.

Die Veränderungen der Drüsen und Lacunen betreffen einmal das Epithel, dann das periglanduläre Gewebe.

Die Veränderungen des Epithels sind in allen Fällen die gleichen.

Das Epithel der Lacunen, eine Fortsetzung des Epithels der Urethra, zeigt Proliferation und schleimige Degeneration der obersten Schichte der Cylinderzellen, Verbreiterung der Ersatzzellenschichte auf mehrere Lagen. Durch diese Zellproliferation im Vereine mit der Verengerung des Lumens durch perilacunäres comprimirendes Infiltrat erscheint das Lumen vieler Lacunen mit abgestossenen proliferirten Zellen ganz erfüllt.

Die Veränderungen des perilacunären Bindegewebes sind bei Besprechung der Veränderungen des subepithelialen Bindegewebes schon besprochen worden. Weder in der Pars anterior noch in der Pars posterior konnte ich eine wesentlichere Localisation des Processes in die Lacunen und Drüsen in dem Sinne eines rein glandulären Processes 
constatiren, so dass das Infiltrat nur um Lacunen und Drüsen, nicht aber im übrigen subepithelialen Bindegewebe localisirt, oder auch nur um Lacunen und Drüsen herum dichter wäre. Stets erscheint das ganze subepitheliale Bindegewebe gleichmässig infiltrirt und die Lacunen und Drüsen zeigen perilacunäre, periglanduläre Infiltration eben nur daram, weilsiein das infiltrirte subepitheliale Bindegewebe eingebettet sind. Ist dieses subepitheliale Infiltrat im ersten Stadium, so vermag es nur Compression des Lumens der Lacunen zu bedingen, wie ich eben erwähnte. Uebergeht aber das Infiltrat in das zweite Stadium der Bindegewebsbildung und Schrumpfung, so führt diese zu Compression und schliesslich völliger Vernichtung der Lacunen (Fall VII).

Was ron dem perilacunären Gewebegilt, gilt a uch von dem periglandulären. Auch die Drüsen zeigen keine isolirte periglanduläre Infiltration, sie sitzen nur im infiltrirten subepithelialen Bindegewebe, zeigen periglanduläre Infiltration, nur soweit das subepitheliale Bindegewebe infiltrirt ist. Und so kommt es, dass eine grössere, tiefer liegende Drüse nur zum Theil, dort, wo sie der Schleimhautoberfläche näher ist, periglanduläre Infiltration zeigen, mit ihrem tieferen Theil nach unten aus dem Infiltrat herausragen und in normalem periglandulären Gewebe sitzen, oder dass in demselben Falle oberflächlich liegende Drüsen periglanduläre Infiltration, tiefer liegende normale Umgebung zeigen können (Fall III, V, VII-X). Kommt das subepitheliale Bindegewebe zur Schwielenbildung und Schrumpfung, so werden die in der Schwiele liegenden Drüsen zerstört (Fall VII). Von Veränderungen des Epithels haben wir nur eine Proliferation, eine Vermehrung des Epithels mit Abstossung zu nennen, die bis zur völligen Erfüllung des Lumens führen kann (Fall I, III-VI, VIII-X). Daneben aber können in dem Lumen der Drüsen, und zwar, wie ich ausdrücklich betone periglandulär völlig normaler Drüsen auch polynucleare Leucocyten (Fall VIII) auftreten, die entweder in geringerer Zahl dem desquamirten Epithel beigemengt sind oder an Menge so sehr überwiegen, dass der Inbalt der Drüse ein rein eitriger er- 
scheint. Desquamativer, desquamativ-eitriger Catarrh mit und ohne periglanduläre Veränderungen sind also die wesentlichen Erscheinungen an den Drüsen der Urethra.

b) Caput gallinaginis. Von den drüsigen Elementen des Caput gallinaginis werden uns Utriculus und die Ductus ejaculatorii später beschäftigen.

Neben diesen aber besitzt das Caput gallinaginis noch zahlreiche Drüsen, die nach dem Typus der Prostatadrüsen gebaut sind, also tubulöse Drüsen mit verzweigten Tubulis, deren bindegewebige Zwischenräume am Durchschnitte als Zotten sich präsentiren und einem Epithel, das aus einer oberen Reihe von palissadenförmig angeordneten Epithelzellen besteht, die bald mehr als Cylinder-, bald mehr als cubisches Epithel imponiren mit einer Reihe von Ersatzzellen.

Auch ron diesen Drüsen gilt, was die periglandulären Veränderungen betrifft, der Hauptsacbe nach das ron den Drüsen der Urethra Gesagte. Die oberflächlichen Drüsen zeigen meist periglanduläre Veränderungen nur insoferne und soweit, als sie in dem subepithelialen Infiltrate sitzen, während die tieferen, ausser Bereich des subepithelialen Infiltrates sitzenden Drüsen keine periglandulären Veränderungen zeigen. Doch gilt das nicht ausnahmslos. So geschieht es, dass das subepitbeliale Infiltrat an den Drüsen um diese herum tiefer herabsteigt, als sonst im Bindegewebe, also die Drüsen dann eine wahre periglanduläre Infiltration darbieten (Fall I, VII). Dieses Infiltrat hat dann immer denselben Charakter wie das subepitheliale Infiltrat, besteht also aus Rundzellen mit bald mehr, bald weniger epitheloiden Zellen, wohl auch Spindelzellen.

Diese periglanduläre, als Fortsetzung des subepithelialen Infiltrates in die Tiefe aufzufassende Infiltration ist aber dann auch inconstant, d. h. an einer Drüse nachzuweisen, während sie an der Nachbardrüse fehit.

Dieses periglanduläre Infiltrat, das insbesondere um die hochgelegenen Drïsen, bei massiger Entwicklung im subepithelialen Gewebe auch dicht und massig erscheint, führt zu Compression der Drüse, Verkleinerung des Lumens bis zur Reduc- 
tion desselben zu capillaren Spalten. Dieses periglanduläre Infiltrat, wenn es als rein periglanduläres Infiltrat, schon ausser Bereich des subepithelialen Infiltrates an der Drüse herunterzieht, kann nun entweder das die Wand der Drüse bildende Bindegewebe gleichmässig durchsetzen, es ist also wirklich periglandular (Fall I, VII) es kann aber sich hauptsächlich in die bindegewebigen Interstitien zwischen den einzelnen Tubulis, in die Zotten localisiren, es ist dann also eigentlich mehr interstitiell. Durcb, diese auf die Zotten, das interacinöse, oder richtige intertubulöse Bindegewebe localisirte Infiltration werden die Zotten verlängert, legen und krümmen sich, ja sie scheinen (Fall II) nach Verlust ihres Epithels verwachsen zu können, wodurch theilweise Abschliessung der Tubuli zu Stande kommt, die Drüse am Durchschnitt einen acinösen Typus gewinnt (Fig. I $c$ ).

Neben diesen periglandulären Veränderungen, aber unabhängig von denselben, $d$. h. sowohl an Drüsen, deren periglanduläres Bindegewebe infiltrirt, als an Drüsen und Drüsenabschnitten, deren periglanduläres Gewebe normal erscheint, kommen endoglanduläre Veränderungen vor. Diese endoglandulären Veränderungen bestehen zunächst in der Mehrzahl der Fälle (Fall I-III, V-VII, X) in Proliferation und Desquamation des Epithels. Dieses abgestossene Epithel, dessen Zellen durch gegenseitige Abplattung meist mehr polygonale oder Spindelform darbieten, erfüllt oft im Vereine mit grossen einzelnstehenden oder zahlreichen kleinen Amyloiden das Lumen der Drüse ganz.

In anderen Fällen (Fall VIII, IX) und zwar wieder völlig unabbängig von periglandulären Veränderungen treten in Drüsen oder Drüsenabschnitten Eiterkörperchen in geringerer oder in dominirender Zahl auf, bis zur Erfüllung des Lumens mit anscheinend rein eitrigem Inhalt.

Dass das subepitheliale Infiltrat, wenn es in Schwielenbildung übergeht, oberflächliche Drüschen ganz zu zerstören vermag, ist wohl klar.

Durch die schwielige Degeneration der oberen Lagen des subepithelialen Bindegewebes in Fall VII, die bandförmig über 
das Caput gallinaginis hinziehende Narbe in Fall X scheinen aber auch die Ausführungsgänge einiger dieser Drüschen obliterirt, denn nur so erklären sich die aus Drüsen hervorgegangenen kleinen Cystchen im Caput gallinaginis, in denen durch Ausdehnung der Tubuli die Zotten völlig verstrichen sind, die Drüse einen kugeligen Hohlraum darstellt, der noch von charakteristischem niederen Cylinderepithel ausgekleidet, im Lumen Epithelzellen und Amyloide birgt.

c) Prostata. Seltener als die Drüsen des Caput gallinaginis nehmen die Drüsen der Prostata an dem Processe theil. Während die Drüsen des Caput gallinaginis nur in eiuem Falle unverändert befunden wurden (Fall IV), war dies bei der Prostata viermal (Fall II, IV, V, VII) der Fall. Auch ist in den Fällen, wo die Drüsen der Prostata am Processe Antheil nahmen, dies nie mit allen Drüsen der Fall, ein Theil, es ist schwer zu entscheiden, ob die Mehr- oder Minderzahl der Drüsen, bleibt stets normal.

Noch viel seltener als am Caput gallinaginis sind an den Drüsen der Prostata periglanduläre Veränderungen, die dann auch als Fortsetzung des subepithelialen Infiltrates längs den Drüsen und deren Gängen entstanden. Diese periglanduläre Infiltration ist nur zweimal zu constatiren (Fall I, VI). Sie ist auch nicht um alle, sondern nur um einen Theil der erkrankten Drüsen nachweisbar.

Das periglanduläre Infiltrat kann, aus mononuclearen Rundzellen und epitheloiden Zellen bestehend, locker und wenig dicht sein (Fall I), es kann aber auch compacter, dichter erscheinen und hïllt dann in Form langgezogener Spindeln angeordnete Schollen gelben Pigmentes, als Reste alter Hämorrhagien (Fall VI).

Unabhängig ron den periglandulären Veränderungen, d. h. auch in einer grossen Zahl von Drüsen, deren Wand völlig normal ist, zeigen sich die bereits bekannten endoglandulären Veränderungen: rein desquamativer Catarrh, Proliferation und Desquamation des Epithels, mit Erfüllung des Drüsenlumen mit polygonalem Epithel und Amyloiden (Fall I, III, X) desquamativ-eitriger bis rein eitriger Catarrh, Erfüllung des Lumens mit desquamirtem Epitbel und 
polynuclearen Leucocyten oder mit letzteren allein in oft sehr bedeutender Menge. Auffällig ist in den Fällen von eitrigem Catarrh die geringe Zahl, ja das völlige Fehlen von Amyloiden.

Dieser entweder rein desquamative oder desquamativeitrige Catarrh ist klinisch von grossem Interesse. Der anatomische Nachweis desselben lehrt uns, dass wir zwei Formen von Prostatorrhoe zu unterscheiden haben, bei deren einer, dem desquamativen Catarrh, das Prostatasecret nur vermehrte Epithelzellen, bei der zweiten, dem eitrigen Catarrh dagegen neben Epithel auch Eiterzellen führt.

Leider belehren uns meine Präparate nicht über den Endausgang des Processes. Da der Process sich nur in der Drüse abspielt, das periglanduläre Gewebe völlig intact zu sein pflegt, ist wohl Uebergang in Abscedirung ausgeschlossen, um so mehr, als wenn periglanduläre. Veränderungen auftreten (Fall I, VI), dieselben mehr zur Bindegewebsbildung und Schrumpfung neigen.

Möglich wäre es, dass zum desquamativen oder desquamativeitrigen Catarrh bei langem Bestand periglanduläre chronische Infiltration mit Ausgang in Schrumpfung hinzutritt, also die Fälle ohne periglanduläre Erkrankung ein früheres, die mit periglandulärer Infiltration ein späteres Stadium des Processes darstellen, der mit Zugrundegehen der erkrankten Drüsen endet.

\section{Utriculus.}

Gleich den übrigen Drüsen des Caput gallinaginis nimmt auch der Utriculus und in analoger Weise an dem chronischen Entzündungsprocesse theil. Auch hier haben wir Veränderungen der Wand und Veränderungen am Epithel zu erwähnen.

Die Veränderungen der Wand sind eine directe Fortsetzung der schon beschriebenen Veränderungen des subepithelialen Bindegewebes, ihnen daher im grossen Ganzen analog. Und so zeigt der Utriculus in einer Reihe von Fällen periglanduläre Infiltration mit Rundzellen und epitheloiden Zellen, die dichter oder mehr locker, entweder den Utriculus nur partiell, im oberen Antheil oder total umgibt. Diese periglanduläre Infil tration umgibt den Utriculus, sitzt in dessen periglandulärem Bindegewebe oder sie ist nur auf dessen Zotten, die binde- 
gewebigen Scheidewände der tubulösen Endbläschen beschränkt. Diese Zotten werden dann durch das Infiltrat verbreitert, verlängert (Fall IV), ja sie scheinen auch verwachsen zu können (Fall II), wodurch Abschluss der Endbläschen und dadurch ein mehr acinöses Aussehen der Drüse erfolgt. Ist das Infiltrat im subepithelialen Bindegewebe schon älter, in schwieliger Umwandlung, dann zeigt das periglanduläre Bindegewebe des Utriculus auch dieselbe Veränderung (Fall V), es ist schwieliger, aber noch reich an mononuclearen, besonders aber Spindelzellen und dieselbe Veränderung zeigt dann auch das Bindegewebe der Zotten.

Auch die Veränderungen des Epithels sind äie den anderen Drüsen analogen. Auch hier handelt es sich, entweder in allen den zahlreichen Tubulis des Utriculus oder nur in einem Theile derselben - während ein anderer Theil normal ist — einmal um rein desquamativen Catarrh, Proliferation und Desquamation des Cylinderepithels, Verbreiterung der Ersatzzellenschichte. Das Lumen der Drüse oder kranken Drüsentheile ist dann mit proliferirtem Epithel und Amyloiden erfïllt (Fall I, II, IV, VI) (Fig. I $d$ ) oder es handelt sich, auch meist wieder nur in einem Theile der Drüsentubuli, um desquamativ-eitrigen bis rein eitrigen Catarrh, das Lumen der betreffenden Tubuli hält Epithelzellen mit Leucocyten oder ietztere allein in oft recht beträchtlicher Menge. Diese Vorgänge im Drüseninneren sind von den periglandulären Vorgängen unabhängig, und so zeigt gerade Fall V, in dem die periglanduläre Infiltration bis zur Bildung schwieligen Bindegewebes vorgeschritten ist, keine Veränderungen des Epithels, während von den beiden Fällen eitrigen Catarrhes in dem einen (Fall VIII) periglanduläre Erkrankung völlig fehlt (Fig. IIe), in dem anderen (Fall IX) nur sehr gering angedeutet ist.

Interessante, mehr secundäre und passive Veränderungen zeigt Fall $\mathrm{X}$. Hier ist die Mündung des Utriculus durch ein über die Kuppe des Caput gallinaginis hinziehendes schwieliges Band, dessen wahrscheinliche Genese wir schon besprochen haben, obliterirt. In Folge des Verschlusses der Mündung bat nun der Utriculus eine sehr bedeutende Dilatation erfihren. De norma auf das Caput gallinaginis beschränkt, reicht er hier 
als blindes Divertikel bis tief in die Prostata zwischen beiden Ductus ejaculatorii verlaufend, hinein. Seine de norma zahlreichen Divertikel, tubulösen Enddrüschen sind verstrichen, er stellt ein gleichmässiges Cavum mit glatter Wand, ovalem Durchschnitt dar. Daneben ist aber ebenso die Wand, als das Epithel krank. Die Wand zeigt in von oben nach unten abnehmender Intensität Infiltration mit kleinen Rundzellen und epitheloiden Zellen, das Epithel zeigt Proliferation der Cylinderzellen, Verbreiterung der Ersatzzellenschichte, das Lumen führt Epithelzellen, Pigmentschollen, Detritus, aber keine Amyloide.

\section{E. Ductus ejaculatorii.}

Die Miterkrankung der Ductus ejaculatorii ist inconstant. So waren dieselben in vier Fällen (Fall III und VI-VIII) völlig normal. In den anderen Fällen aber zeigten die Ductus ejaculatorii sehr interessante und für die Pathologie des Sexuallebens sehr instructive und bedeutungsvolle Veränderungen.

Diese Veränderungen spielen sich vorwiegend in der Wand der Ductus ejaculatorii ab, sind also, wenn ich so sagen. darf, vorwiegend periglandulär.

So sehen wir: gleich im Falle I (Fig. Ic), in dem das subepitheliale Gewebe von Urethra und Caput gallinaginis Sitz eines dichten und tiefgreifenden theilweise necrotisirten Rundzelleninfiltrates ist, wie die Mündung beider Ductus ejaculatorii, respective deren kurzer Verlauf durch das Infiltrat hindurch wesentliche Veränderungen aufweist. Das Infiltrat comprimirt das Endstiuck der D. ejaculatorii auf einən schmalen Spalt. Die Wand desselben wird ausschliesslich von Infiltrat gebildet, hat ihr Epithel eingebüsst.

Aber auch vor dem Eintritte in das Infiltrat zeigen beide Ductus ejaculatorii eine Wanderkrankung, indem das Infiltrat des subepithelialen Bindegewebes sich in der Wand der Ductus ejculatorii herabzieht, dieselben als schlauchförmiges Infiltrat während ibres Verlaufes durch dis Caput gallinaginis und die Prostata umgibt. Wohl nimmt dieses Infiltrat nach der Tiefe zu allmälig an Dichte $a b$, ist aber doch bis tief in das Gewebe der Prostata zu verfolgen. Nachdem die Ductus ejaculatorii keine Canäle mit glatter Wand sind, sondern in deren Wand 
zahlreiche divertikelförmige vom gleichen Epithel wie der Gang überzogene einfach oder mehrfach dichotomisch verzweigte Drüsentubuli sitzen, so sitzt das Infiltrat nicht nur periglandulär, sondern auch iuterstitiell, in dem sich auf Querschnitten als Zotten producirenden, von Epithel überzogenen Bindegewebe. Analoge nur geringgradigere Veränderungen der Wand, Infiltration mit Rundzellen, epitheloiden, theilweise auch Spindelzellen zeigen die Fälle II und IV. Nur ist hier das Infiltrat weniger dicht, begleitet die Ductus ejaculatorii nur innerhalb ihres Verlaufes durch das Caput gallinaginis, ist anfangs in dem oben angedeuteten Sinne sowohl periglandulär als interstitiell. Das periglanduläre Infiltrat hört früher auf, so dass es schliesslich nur als interstitiell zurückbleibt, um nach unten $\mathrm{zu}$ immer lockerer zu werden und aufzuhören.

War in diesen drei Fällen das die Ductus ejaculatorii einschliessende Infiltrat im ersten Stadium, im Stadium der Rundzelleninfiltration, so zeigt uns Fall V ein schönes Beispiel schwieliger Degeneration der Wand beider Ductus ejaculatorii (Fig. III $\left.d, d^{\prime}, e\right)$. Es handelt sich in diesem Falle um Infiltration des subepithelialen Bindegewebes, die schon weit am Wege schwieliger Degeneration vorgeschritten ist. Also strafferes, derberes, an Spindelzellen reiches, aber auch noch von Rundzellen durchsetztes Bindegewebe. Diese Veränderung des Bindegewebes setzt sich aus dem subepithelialen Bindegewebe einmal, wie bereits erwähnt, längs des Utriculus fort, sie setzt sich dann aber auch längs beider Ductus ejaculatorii fort, während deren Verlauf durch das Caput gallinaginis und den Anfangstheil der Prostata.

Das Epithel der Ductus ejaculatorii zeigt in diesen Fällen keine wesentlichen Veränderungen. Auffällig ist höchstens der Reichthum von Pigmentschollen in Fall II (26 Jahre alter Mann).

Aber ein weiterer auffälliger Befund in zwei Fällen (Fall II, V) verdient Beachtung.

Wie erwähnt, besitzt die Wand der Ductus ejaculatorii zahlreiche Divertikel, in denen tubulöse, oft mehrfach fingerförmig getheilte, oft sehr lange Drüsen sitzen. Alle diese Divertikel münden in spitzem Winkel in den Ductus ejaculatorius und sehen mit ihrer Mündung nach dem Caput gallinaginis, 
nach der Mündung der Ductus ejaculatorii hin. In den genannten zwei Fällen (Fig. III $d, d^{\prime}$ ) nun finden sich die meisten dieser Divertikel sowohl innerhalb des Caput gallinaginis als auch während des ganzen Verlaufes durch die Prostata dicht erfiillt mit den Elementen des Sperma, insbesondere reichlichen, in dichten Haufen die Tubuli voll füllenden Spermatozoen. Bei der Richtung aller dieser Divertikel nach der Mündung der Ductus ejaculatorii hin, kann das Sperma in diese Divertikel nur durch rückläufige, retroperistaltische Bewegung hineingelangen. Nun zeigt Fall II Verengerung der Mündung der Ductus ejaculatorii durch subepitheliales Rundzelleninfiltrat am Caput gallinaginis, in Fall IV zeigt der Verlauf der D. ejaculatorii durch das Caput gallinaginis Starrheit der Wand durch in schwieliger Degeneration begriffene Infiltration derselben. Es läge nahe, diese beide, die Ejaculation gewiss behindernde Momente für die rückläufige Bewegung der Sperma verantwortlich zu machen.

Ébenso interessant sind die mehr passiven Veränderungen der Ductus ejaculatorii durch narbige Obliteration ihrer Mündung, wie diese in Fall IX der linke, in Fall $\mathrm{X}$ beide Ductus ejaculatorii zeigen. Von der narbigen Verschliessung ab sind die Ductus ejaculatorii sehr bedeutend erweitert, die Divertikel und Buchten der Wand ganz oder grösstentheils verstrichen. In Fall X (Fig. V) ist nebenbei lockere Infiltration der Wand mit mononuclearen Rundzellen nachweisbar, die Blutgefässe des subepithelialen Bindegewebes zeigen auffällige Dilatation, an zahlreichen Stellen ist es zur Zerreissung der Blutgefässe, zu capillaren subepithelialen Hämorrhagien gekommen, die das Epithel in weitem Umkreis unterwïhlen, abheben, zerreissen, wodurch auch Blutkörperchen in das Lumen gelangen. In diesem finden sich nebenbei noch Epithelzellen, Pigmentschollen, in Fall IX auch polynucleare Leucocyten.

Dass diese narbige Verschliessung der linken D. ejaculatorii zu Oligospermie, beider Ductus ejaculatorii aber zu Aspermatismus bei den betreffenden. Individuen fuihrte, ist klar. Die capillaren Blutungen älteren und neueren Datums in Fall $X$ mögen wohl bei ejaculatorischen Contractionen der Samenbläschen bei Coitus oder Pollution entstanden sein. 


\section{Schlussfolgerungen.}

1. Der chronische Tripper der Pars posterior stellt sich uns gleich dem der Pars anterior im Wesentlichen dar als eine chronische Entzündung des subepithelialen Bindegewebes, die zwei Stadien durchläuft. Ein erstes Stadium der Infiltration, der Bindegewebsneubildung, ein zweites Stadium der Schrumpfung: und Schwielenbildung.

2. In die obersten Schichten des subepithelialen Bindegewebes localisirt, verläuft der Process auch der Hauptsache nach in diesen. Doch vermag die chronische Bindegewebsneubildung sich an und um Drüsen, um den Utriculus, die Ductus ejaculatorii in bedeutende Tiefe fortzusetzen und indem sie auch hier in das zweite Stadium der Schrumpfung übergeht, wesentliche Veränderungen der Wand der genannten Drüsen und Drüsenausführungsgänge zu bedingen.

3. Neben diesen für den Process essentiellen Veränderungen haben wir noch zwei weitere Reihen von Erscheinungen zu nennen, die sich theils als complicatorische, theils als consecutive darstellen.

4. Als complicatorisch ist aufzufassen die Erkrankung der Drüsen der Urethra, des Caput gallinaginis, der Prostata, die wieder theils als rein desquamativer, theils als desquamativeitriger Catarrh abläuft. Ebenso die catarrhalische Erkrankung der Schleimhaut der Urethra.

5. Die consecutiven Erscheinungen sind bedingt durch die im subepithelialen Bindegewebe erfolgende Schwielenbildung. Hierher gehört die Umwandlung des Cylinderepithels der Urethra und des Caput gallinaginis in Plattenepithel, die Zerstörung der Lacunen und oberflächlichen Drüsen, die Obliteration der Mündung des Utriculus und der Ductus ejaculatorii.

Zum Schlusse gelangt, ist es mir eine angenehme Pflicht, Herrn Professor Weichselbaum für seine Liberalität und weitgehende Unterstützung auch an dieser Stelle herzlichst. Dank zu sagen. 


\section{Erklärung der Abbildungen auf Taf. I-III.}

Fig. I. (Fall I.) Querschnitt durch das Caput gallinaginis. a) Dichte kleinzellige Infiltration des subepithelialen Bindegewebes. b) Oberflächliche Heerde inselförmiger Necrose. c) Ductus ejaculatorius, d) Utriculus, dessen Epithel in catarrbalischer Proliferation und Desquamation. e) Prostatische Drüse. Das interstitielle Gewebe derselben kleinzellig infiltrirt, zahlreiche Tubuli durch Verwachsung der Zotten des intertubulösen Bindegewebes abgeschlossen, daher die Drüse den acinösen ähnlichen Bau zeigt.

Fig. II. (Fall VIII.) Caput gallinaginis. a) Proliferirendes Plattenepithel. b) Subepitheliale Infiltration, aus mononuclearen Rundzellen und polynuclearen Leueocyten bestehend. c) Utriculus, in dessen Inhalt reichlich proliferirtes, desquamirtes Epithel und polynucleare Leucocyten (desquamativ-eitriger Catarrh).

Fig. III. (Fall V.) Caput gallinaginis. a) Plattenepithel. b) Subepitheliales Bindegewebe, schwielig reich an Spindelzelien. c) Utriculus. $d d^{\prime}$ ) Beide ductus ejaculatorii, dieselben und ihre Divertikel mit Sperma erfüllt. e) Züge schwieligen Bindegewebes, die die Wand des Utriculus and beider Ductus ejaculatorii bilden.

Fig. IV. (Fall IX.) Caput gallinaginis. a) Vielschichtiges Plattenepithel. b) Narbe, die Hälfte des Caput gallinaginis einnebmend. c) Drüsenreste.

Fig. V. (Fall X.) Ductus ejaculatorius, an der Mündung obliterirt, sein Lumen dilatirt. a) Dilatirtes Lumen, erfüllt mit Detritus, Epithelzellen, Blntkörperchen. b) Epithel des Ductus ejaculatorius. c) Dilatirte Blutgefässe. d) Sủepitheliale Hämorrhagien. 


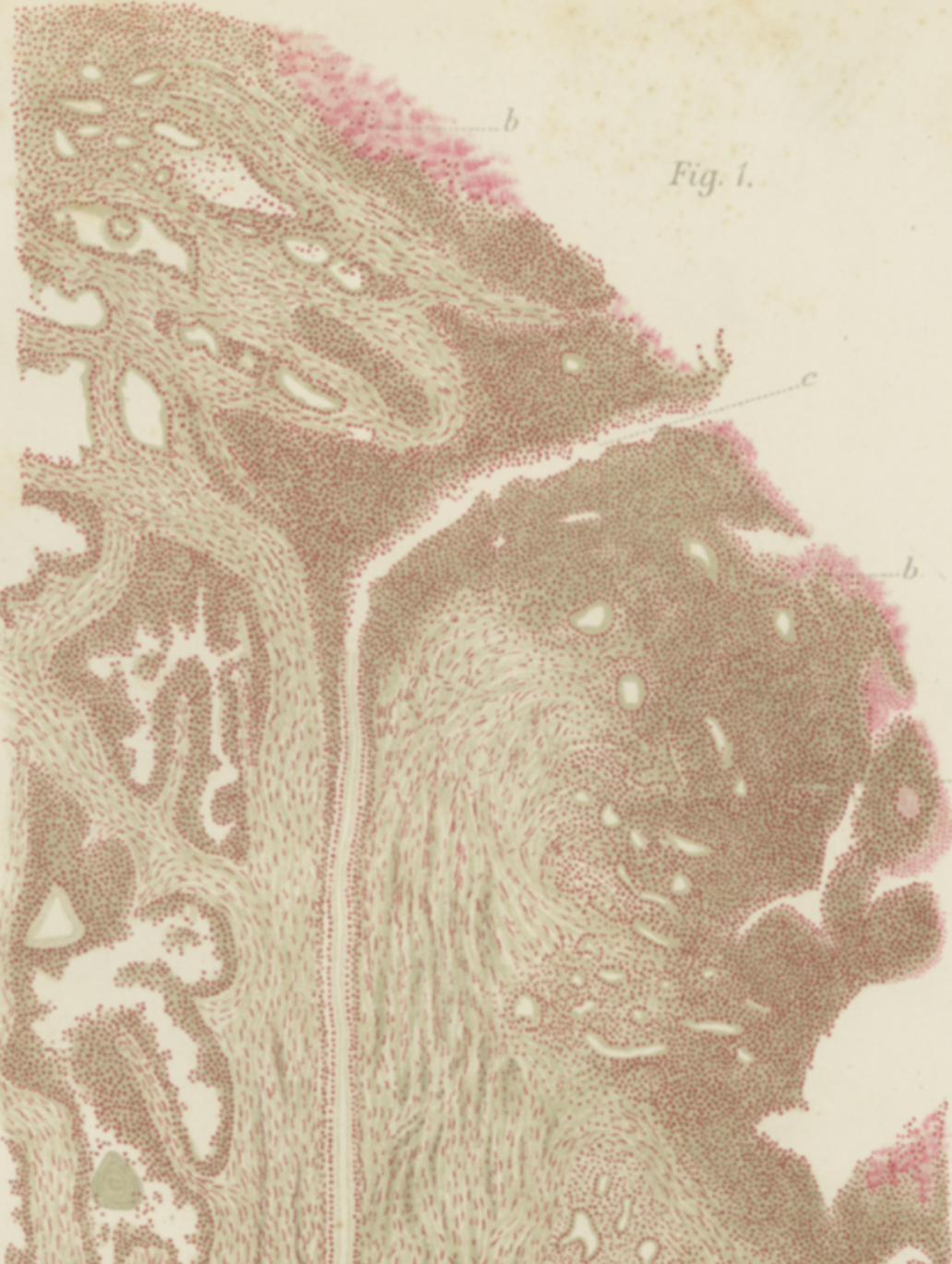

6. 3y, w. 3. 稳

H.

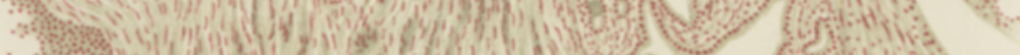

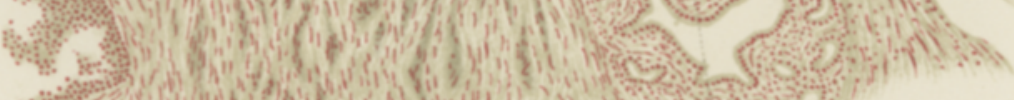

ed 

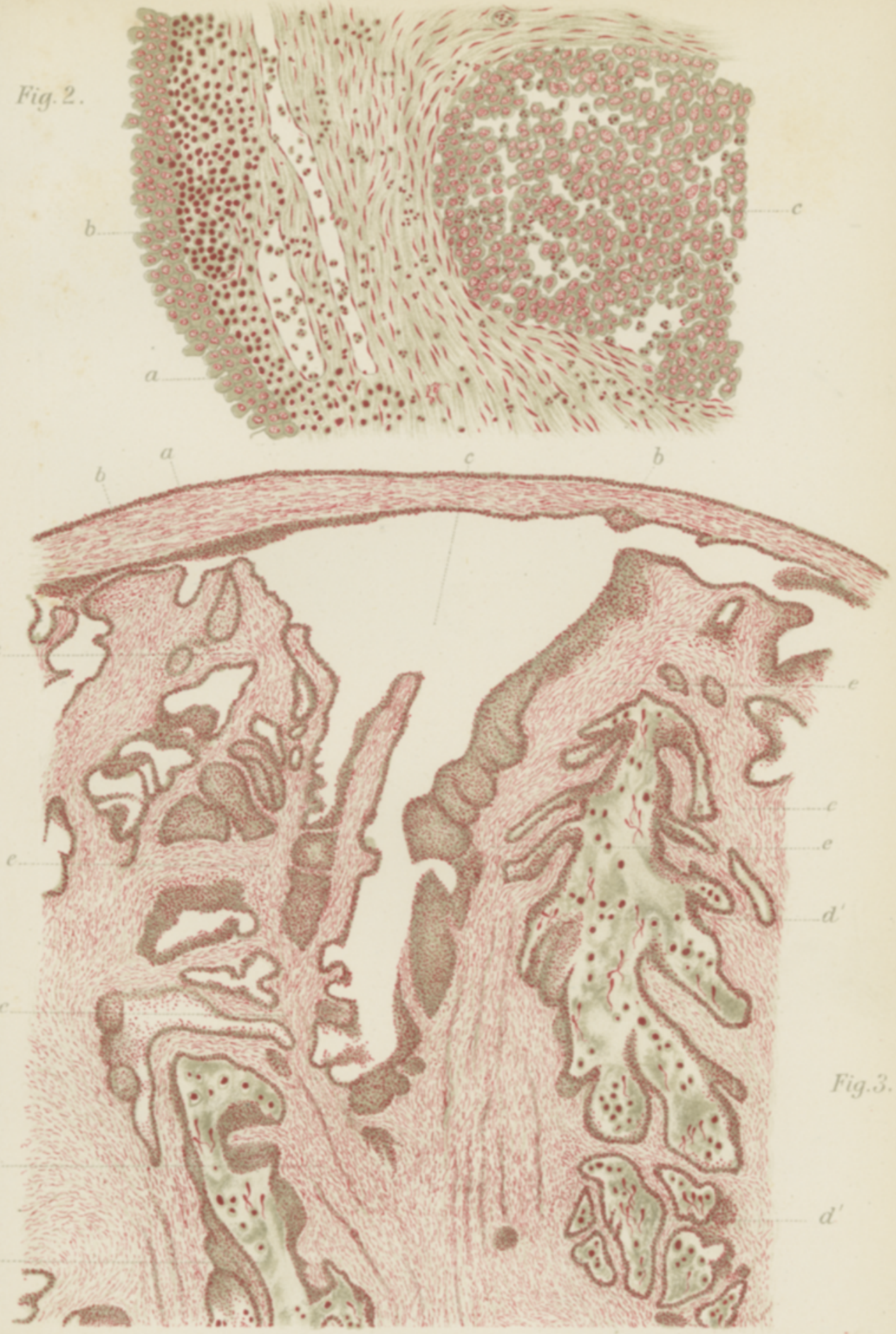


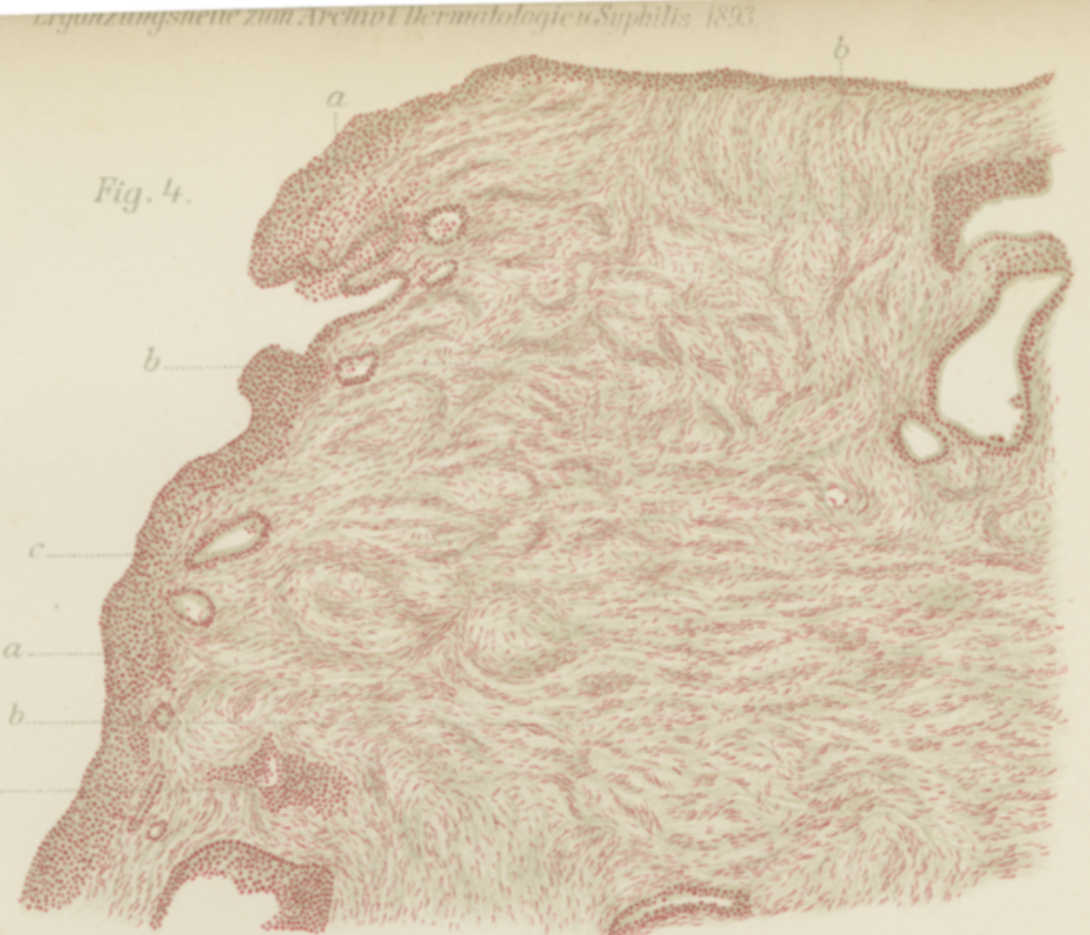

